\title{
Recurrent emergence of carbapenem resistance in Klebsiella pneumoniae mediated by an inhibitory ompK36 mRNA secondary structure
}

Authors: Joshua L. C. Wong ${ }^{1,2}$, Sophia David ${ }^{\dagger}$, Julia Sanchez-Garrido ${ }^{1}$, Jia Z. Woo ${ }^{4}$,

Wen Wen Low ${ }^{1}$, Fabio Morecchiato ${ }^{5}$, Tommaso Giani ${ }^{5,6}$, Gian Maria Rossolini ${ }^{5,6}$, Stephen J. Brett ${ }^{2}$, Abigail Clements ${ }^{1}$, David M Aanensen ${ }^{3}$, Silvi Rouskin ${ }^{4}$ and $\underline{\text { Gad }}$ Frankel $^{1}$

t These authors contributed equally to this work.

Affiliations:

1. Centre for Molecular Bacteriology and Infection, Department of Life Sciences, Imperial College London, London, United Kingdom.

2. Department of Surgery and Cancer, Section of Anaesthetics, Pain Medicine and Intensive Care, Imperial College London, London, United Kingdom.

3. Centre for Genomic Pathogen Surveillance, Big Data Institute, University of Oxford, United Kingdom.

4. Department of Microbiology, Harvard Medical School, Boston, MA, United States of America.

5. Department of Experimental and Clinical Medicine, University of Florence, Florence, Italy.

6. Clinical Microbiology and Virology Unit, Careggi University Hospital, Florence, Italy.

Corresponding author: Gad Frankel g.frankel@imperial.ac.uk 


\section{$1 \quad$ Abstract}

2 Outer membrane porins in Gram-negative bacteria facilitate antibiotic influx. In Klebsiella

3 pneumoniae $(\mathrm{KP})$, modifications in the porin OmpK36 are implicated in increasing

4 resistance to carbapenems. Analysis of large KP genome collections, encompassing

5 major healthcare-associated clones, revealed the recurrent emergence of a synonymous

6 cytosine to thymine transition at position $25(25 c>t)$ in ompK36. We show that the $25 c>t$

7 transition increases carbapenem resistance through depletion of OmpK36 from the outer

8 membrane. The mutation attenuates $\mathrm{KP}$ in a murine pneumonia model, which accounts

9 for its limited clonal expansion observed by phylogenetic analysis. However, in the

10 context of carbapenem treatment, the $25 c>t$ transition tips the balance towards treatment

11 failure, thus accounting for its recurrent emergence. Mechanistically, the $25 c>t$ transition

12 mediates an intramolecular mRNA interaction between a uracil encoded by $25 \mathrm{t}$ and the

13 first adenine within the Shine-Dalgarno sequence. This specific interaction leads to the

14 formation of an RNA stem structure, which obscures the ribosomal binding site thus

15 disrupting translation. While mutations reducing OmpK36 expression via transcriptional

16 silencing are known, we uniquely demonstrate the repeated selection of a synonymous

17 ompK36 mutation mediating translational suppression in response to antibiotic pressure. 


\section{Introduction}

Classical outer membrane (OM) porins in Gram-negative bacteria enable non-specific bidirectional diffusion between the periplasm and extracellular environment (Achouak et al., 2001). This has been exploited in antibacterial chemotherapy as porins act as the key entry point for clinically important classes of antibiotics across the otherwise impermeable OM. However, porin modifications that restrict antibiotic entry have evolved in response to this selective pressure (Vergalli et al., 2020), contributing to the rising global burden of resistant bacterial infections, especially among species of Enterobacteriaceae (Bajaj et al., 2016; Dé et al., 2001; Wong et al., 2019; Fajardo-Lubián et al., 2019; Tran et al., 2010).

Klebsiella pneumoniae (KP) is one of the most clinically significant members of the Enterobacteriaceae family and a leading cause of healthcare-associated infections worldwide (Cassini et al., 2019; Vincent et al., 2020). The majority of resistant KP infections are caused by "high-risk" clonal lineages, including sequence types (ST) 258 and 512, which form a dominant clone associated with the KP carbapenemase (KPC) gene (David et al., 2019). Together with plasmid-encoded carbapenemases, modifications to the major OM porins OmpK35 and OmpK36 play a critical role in mediating KP resistance to carbapenems, a class of antibiotics that is vital for the treatment of severe infections. Resistance-associated modifications broadly fall into those that structurally alter the porin channel and those that abolish or reduce OmpK36 expression.

Structural alterations in OmpK36 are mediated by amino acid insertions into a region of the porin called loop 3 (L3). These insertions narrow the luminal diameter and restrict substrate diffusion, including antibiotics (Wong et al., 2019; Fajardo-Lubián et al., 2019). 
L3 insertions are relatively prevalent among clinical KP isolates, having been found among $12.3 \%(192 / 1557)$ of isolates from a diverse public genome collection (FajardoLubián et al., 2019). We previously showed that the most common L3 insertion, a diamino acid insertion (Glycine-Aspartate, GD), results in a 16-fold increase in minimum inhibitory concentration (MIC) to meropenem (Wong et al., 2019).

In addition to structural modifications, carbapenem resistance is also achieved by absent or reduced OmpK36 expression. Absent expression, which can be achieved by gene truncation, results in high levels of resistance but comes at a significant in vivo fitness cost (Wong et al., 2019; Fajardo-Lubián et al., 2019; Tsai et al., 2011). Reduced OmpK36 expression has been reported to occur by multiple mechanisms that all result from transcriptional silencing, including ompK36 promoter disruption by insertion sequence (IS) elements (Clancy et al., 2013), loss-of-function mutations in kvrA (a transcriptional repressor that controls capsule production) (Dulyayangkul et al., 2020) and mutations in hfq (a regulatory RNA binding protein) (Chiang et al., 2011). However, the effects on virulence resulting from reduced OmpK36 expression are poorly understood. Moreover, the prevalence and clinical significance of these mechanisms remain unknown as the mutations identified to date were either restricted to clinical isolates from a single centre (promoter insertion) or evolved during in vitro selection or genetic deletion experiments (kvrA and $h f q)$.

Here we identify and describe a carbapenem resistance mechanism that has been repeatedly employed by clinical $\mathrm{KP}$ isolates to post-transcriptionally alter OmpK36 abundance via synonymous mutations in the open reading frame (ORF). Our study spans the identification of one such key mutation $(25 \mathrm{c}>\mathrm{t})$ in $\mathrm{ompK} 36$ through large-scale bioinformatic approaches, the assessment of its effects on carbapenem susceptibility and 
66 virulence using murine pneumonia models, and finally the determination of the precise

67 molecular mechanism linking synonymous SNPs with protein abundance. In particular,

68 we show that the $25 c>t$ mutation results in the formation of a stem structure in the ompK36

69 mRNA that obscures the Shine Dalgarno sequence (SDS), reduces OmpK36 translation

70 and abundance, and increases carbapenem resistance as a consequence. This work

71 provides the first example of the functional use of these inhibitory mRNA structures in

72 regulating protein expression during bacterial adaptation, and characterises a novel,

73 clinically-important resistance mechanism in KP. 


\section{Results}

\section{Recurrent emergence of a synonymous 25c>t ompK36 mutation}

We curated and analysed a collection of 1450 public KP genomes belonging to the major healthcare-associated clone composed of STs 258, 512 and other closely related derivatives (https://microreact.org/project/1vWbaqARPRNc55n4yfdLyQ-ompk36; Table S1). The collection comprises isolates gathered between 2003 and 2018, largely in the Americas, Europe and the Middle East, where rampant spread of ST258/512 in healthcare institutions has been reported (Adler et al., 2017; Giakkoupi et al., 2011; Giani et al., 2013; Kitchel et al., 2009; Rojas et al., 2017). We unambiguously identified the ompK36 gene in $98.1 \%(1422 / 1450)$ of the genomes. Among these, the gene was intact in $99.3 \%(1412 / 1422)$. Using the intact ompK36 sequences, we inferred how each position in this gene has evolved across the ST258/512 population. First, we constructed a phylogeny of the collection using all vertically inherited single nucleotide polymorphisms (SNPs) from a core genome alignment, with recombined regions excluded, to provide an accurate representation of the ancestral relationships between isolates (Figure 1A). We then mapped the variation in ompK36 onto this phylogeny and predicted the ancestral states of each position in the gene across the tree using a maximum parsimony approach. While $83.4 \%$ of nucleotide positions are entirely conserved across ompK36, we found two regions with an increased number of base changes (Figure 1B). As anticipated, the largest number of changes occurred in the loop 3 (L3) region comprising amino acid insertions (GD, TD, D and N) (24 occurrences) as well as their reversions (i.e. deletions) (25 occurrences). However, further to this, we observed ten base changes at position 25 , all consisting of a synonymous c>t transition (ㄷTG (leucine)->ITG (leucine)). Despite this mutation maintaining the identical amino-acid translation in the Sec-dependent signal 
sequence (Figure $2 A \& B$ ), its high frequency of emergence suggests that it has undergone positive selection. The majority of isolates with the $25 \mathrm{c}>\mathrm{t}$ mutation are sporadically distributed across the core genome phylogeny and occur as singletons or clusters of only two isolates (11/18) (Figure 1A). The remaining seven isolates, collected between 2009 and 2013 from multiple healthcare institutions in the USA, form a monophyletic cluster, indicative of a clonal expansion. We noted that these seven isolates encode an additional synonymous $24 \mathrm{c}>\mathrm{t}$ mutation in the preceding codon ((CT $\underline{\mathrm{C}}$ (leucine)->CTI (leucine)) (Figure 2B).

We next searched for the $25 \mathrm{c}>\mathrm{t}$ mutation in a large, geographically diverse collection of 16,086 KP genomes (https://pathogen.watch/genomes/all?genusld=570\&speciesld=573;

Table S2) to establish its wider prevalence among sequenced isolates and presence in other (i.e. non-ST258/512) clonal lineages. Among the 14,888 isolates encoding an intact ompK36 gene, we identified the $25 \mathrm{c}>\mathrm{t}$ mutation in $2.5 \%(376 / 14,888)$, which includes isolates from 39 STs and 25 countries. Over half $(51.3 \%$; 193/376) belong to ST258 while many also belong to other globally-important multi-drug resistant clones (e.g. ST15, 19.9\%; ST13, 4.5\%; ST11, 2.7\%). We observed the 24c>t mutation in 1.1\% $(161 / 14,888)$ of genomes from this collection, and only ever in combination with the $25 \mathrm{c}>\mathrm{t}$ mutation. Moreover, we also noted that $93.2 \%(150 / 161)$ of ompK36 sequences containing the $24 \& 25 c>t$ mutations had L3 insertions compared to only $1.9 \%(4 / 215)$ of those with $25 c>t$ only.

\section{The $25 c>t$ mutation increases meropenem resistance}

We modelled the effect of the $25 \mathrm{c}>\mathrm{t}$ mutation (ompK36 $\mathrm{WT}_{(25 \mathrm{c}>\mathrm{t})}$ ) on meropenem resistance by introducing the mutation into ompK36wT of our laboratory KP strain ICC8001 (Wong et 
121 al., 2019) (see Table 1 for list of ompK36 variants and strains used in this study). We

122 additionally deleted ompK35, as this porin gene is truncated in $99.8 \%$ of the ST258/512

123 genomes, and introduced the KPC-2 carbapenemase (on a pKpQIL-like plasmid) given

124 also the strong association of KPC genes with the lineage (Bowers et al., 2015). We found

125 that expression of ompK36 $\mathrm{WT}(25 \mathrm{c}>\mathrm{t})$ results in a meropenem minimum inhibitory

126 concentration (MIC) of $64 \mathrm{mg} / \mathrm{L}$, 8-fold higher than the resistance breakpoint of $8 \mathrm{mg} / \mathrm{L}$

127 (EUCAST, 2021) (Figure 2C). This MIC is also four-fold higher than that seen in KP expressing ompK36 ${ }_{\mathrm{WT}}(16 \mathrm{mg} / \mathrm{L})$, albeit lower than the very high resistance observed when ompK36 was deleted $(\Delta o m p K 36,512 \mathrm{mg} / \mathrm{L})$, indicating that the $25 \mathrm{c}>\mathrm{t}$ is a carbapenem resistance mutation.

131 We also investigated the level of resistance conferred by the combination of the $24 \mathrm{c}>\mathrm{t}$ and $25 c>$ t mutations. While ICC8001 did not tolerate the genomic insertion of the double $24 \& 25 c>t$ mutations on a WT ompK36 background, we found that they were tolerated 134 when introduced in combination with a L3 GD pore-constricting insertion

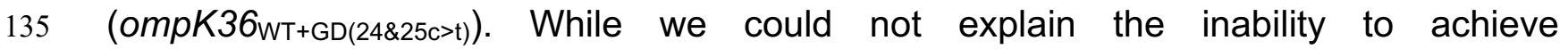
ompK36 ${ }_{\mathrm{WT}}(24 \& 25 \mathrm{c}>\mathrm{t})$ introduction, it nevertheless reflects the strong association between the combined $24 \& 25 c>t$ mutations and L3 insertions observed in our genomic analysis.

138 Broth microdilution testing of the strain with ompK36 $\mathrm{WT}+\mathrm{GD}(24 \& 25 \mathrm{c}>\mathrm{t})$, together with strains

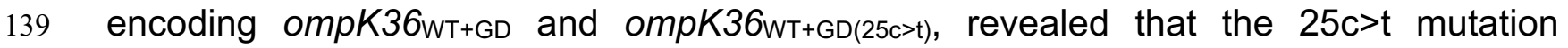

140 increased the meropenem MIC of the L3 GD insertion strain from $64 \mathrm{mg} / \mathrm{L}$ (WT+GD) to $141256 \mathrm{mg} / \mathrm{L}(\mathrm{WT}+\mathrm{GD}(25 \mathrm{c}>\mathrm{t}))$ and that the double $24 \& 25 \mathrm{c}>$ t mutations partially reversed this 142 phenotype, with an MIC of 128mg/L (WT+GD(24\&25c>t)) (Figure 2C). 


\section{failure}

146

147

148

149

150

151

152

153

154

155

156

157

158

The general absence of clonal expansion among isolates with the single $25 \mathrm{c}>t$ substitution, despite the frequent emergence of this mutation, suggests that it may have a fitness cost that impedes onward transmission. We tested this hypothesis in vivo by infecting mice with $250 \mathrm{CFU}$ of $\mathrm{KP}$ encoding either ompK36 $6_{\mathrm{WT}}$ (KP36wT) or ompK36 ${ }_{W T(25 c>t)}\left(K^{2} 366_{W T(25 c>t)}\right)($ Table 1). Inoculation with a strain encoding an ompK36 deletion (KP $\Delta 36)$ and PBS were used as controls (Figure $3 \mathrm{~A}$ ). At $48 \mathrm{~h}$ post infection, KP36wT, but not KP36 ${ }_{W T}(25 c>t)$, induced significant weight loss compared to uninfected (PBS) mice or mice infected with KP $\Delta 36$, Figure 3B). Bacterial counts in the lungs showed a trend to higher burdens in mice infected with $\mathrm{KP} 36_{\mathrm{wT}}$ than with $\mathrm{KP} 36_{\mathrm{wT}}(25 \mathrm{c}>\mathrm{t})$, which reached significance in the blood (Figure 3C\&D). KP $\Delta 36$ demonstrated defects in bacterial survival and proliferation in the host, with significantly lower and/or absent burdens in the lungs and blood (Figure 3C\&D). Interferon gamma (IFNY) is a key cytokine in the defense against KP and measurement of its levels in serum provides a readout of acute inflammatory responses (Moore et al. 2002); following infection with KP36wT, IFNY responses were significantly higher compared to infection with KP36wT(25c>t) (Figure 3E). Taken altogether, these findings show that the $25 \mathrm{c}>\mathrm{t}$ transition attenuates KP in vivo with reduced bacteraemia and the resulting induction of a diminished host pro-inflammatory immune response.

We next evaluated if ompK36 ${ }_{\mathrm{WT}(25 \mathrm{c}>\mathrm{t})}$ provides an advantage compared with ompK36 ${ }_{\mathrm{WT}}$ in the context of meropenem therapy, which would explain the recurrent emergence of this mutation despite its associated fitness cost. We infected mice with either KP36wT or

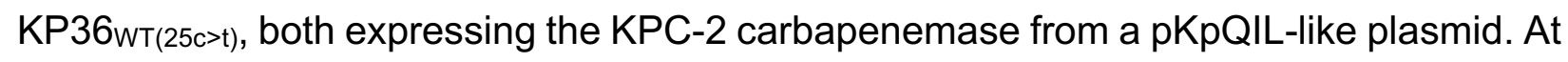


$24 \mathrm{~h}$ mice either received meropenem (100 $\mathrm{mg} / \mathrm{kg}$ dose) or vehicle control (water) by intraperitoneal injection at six hourly intervals for $24 \mathrm{~h}$ (Figure 3F). The experiment was stopped $3 \mathrm{~h}$ after the last injection. No significant differences in body weight were observed following infection by either strain with or without meropenem therapy (Figure $3 G)$, consistent with fluid resuscitation provided by meropenem or vehicle administration. However, significant reductions in the bacterial burdens in the lungs and blood were seen following meropenem treatment of KP36 ${ }_{W T}+K P C-2$ but not KP36 ${ }_{W T}(25 c>t)+K P C-2$ infection (Figure $3 \mathrm{H} \& \mathrm{I}$ ). Furthermore, while the level of serum IFNY was significantly decreased when $\mathrm{KP} 36_{\mathrm{WT}}+\mathrm{KPC}-2$ infection was treated with meropenem (Figure $3 \mathrm{~J}$ ), no significant differences were observed between antibiotic or mock treated KP36 ${ }_{\mathrm{wT}(25 \mathrm{c}>\mathrm{t})}+\mathrm{KPC}-2$ infected mice. These in vivo results show whilst the $25 \mathrm{c}>\mathrm{t}$ mutation in ompK36 attenuates $\mathrm{KP}$, it provides a selective advantage during carbapenem therapy.

\section{The 25c>t mutation reduces OmpK36 abundance}

We next aimed to understand how the $25 \mathrm{c}>\mathrm{t}$ mutation mediates an increase in meropenem resistance and an associated decrease in virulence. Since the mutation results in both carbapenem resistance and virulence phenotypes between those observed in normal (ompK36 $\left.{ }_{\mathrm{WT}}\right)$ and absent ( $\triangle \mathrm{ompK36)}$ expression, we hypothesized that these phenotypic changes were due to reduced OmpK36 abundance in the OM. Indeed, analysis of isolated OM fractions by SDS-PAGE and Coomassie staining revealed that the strain encoding $\mathrm{ompK} 36_{\mathrm{WT}(25 c>t)}$ exhibited a substantially reduced abundance of OmpK36 compared to that with ompK36wt (Figure 2D), validating this hypothesis. In line with the meropenem MIC results, we also found that this pattern is 


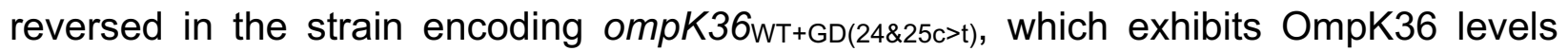
similar to the strain encoding ompK36 $\mathrm{WT}$ (Figure 2D).

\section{Reduced OmpK36 abundance via the 25c>t mutation occurs independently of codon bias}

We sought to describe the precise molecular mechanism linking the synonymous $25 c>t$ mutation to decreased OmpK36 abundance. We first explored whether reduced OmpK36 abundance is due to a decreased translation rate, resulting from a change in codon from the commonly-used CTG to the rarely-used TTG at amino acid position 9 (Leu9). Overall, $64.9 \%$ of leucine residues in the ICC 8001 genome are encoded by CTG and $5.9 \%$ encoded by TTG (Figure 4A); inspection of all the ST258/512 genomes confirmed a similar bias (CTG range: 57.0-65.1\%; TTG range: 5.9-9.3\%).

To investigate this, we generated a fluorescent ICC8001 reporter, in which the ompK36 ORF was replaced with the ompK36 signal sequence fused to sfGFP (Figure 4B). This reporter maintains the upstream promoter and regulatory regions at the monocistronic ompK36 locus. Using sfGFP fluorescence as a proxy for protein expression, we found that the signal sequence containing the $25 \mathrm{c}>\mathrm{t}$ mutation (TTG codon) significantly reduced expression compared to that with the WT sequence (CTG codon) (Figure 4C). This finding is consistent with the reduced OmpK36 abundance observed in the strain encoding ompK36 ${ }_{\mathrm{WT}(25 \mathrm{c}>\mathrm{t})}($ Figure 2D). We then used the CTC leucine codon located at amino acid position eight (Leu8) to test the impact of codon usage on expression. We generated synonymous mutants where Leu8 was encoded by either of the alternative CTG (common) or TTG (rare) codons. Both Leu8 CTG and TTG codons resulted in similar sfGFP expression, which was significantly higher than with the CTC codon found in 


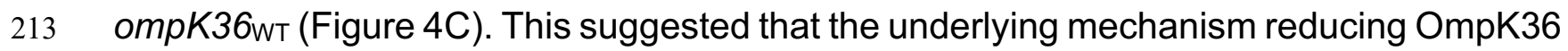

214 expression via the $25 c>t$ mutation is not related to codon usage limiting the efficiency of

215 translation elongation.

216 Reduced OmpK36 abundance is mediated by an interaction between the ORF and 217 5'UTR

218 Mutagenesis studies in $E$. coli have suggested that specific intramolecular RNA 219 interactions between nucleotides in the 5' end of an ORF and the upstream 5'UTR region can result in secondary structures that occlude the SDS, blocking ribosomal access

221 (Kudla et al., 2009; Bhattacharyya et al., 2018). This disrupts the initiation of translation, 222 reducing protein expression, and in turn results in mRNA degradation as transcription and 223 translation are tightly coupled (Bhattacharyya et al., 2018). However, mutations reducing translation efficiency have not been identified as a naturally occurring mechanism tuning protein expression in adaptation to a host or environmental pressure. Nonetheless, we hypothesized that the reduced abundance of OmpK36WT(25c>t) may be due to an inhibitory mRNA secondary structure, mediated by a specific a/u base-pairing occurring between an adenine within the 5' UTR of ompK36 (Figure 4D) and the uracil encoded by $25 \mathrm{t}$. We started by confirming the lower abundance of ompK36 mRNA transcripts in the 25c>t mutant compared to WT by qRT-pCR (Figure 4E), a finding consistent with increased mRNA degradation. We then individually mutated each adenine to a thymine (starting from the end of the -10 box/transcription start site) in the 5'UTR of the sfGFP reporter 233 containing the $25 \mathrm{c}>\mathrm{t}$ mutation in the signal sequence. We hypothesized that the disruption 234 of any base interaction between $25 u$ and a position in the 5'UTR would be evidenced by 235 increased sfGFP expression. No significant changes in fluorescence were observed in 236 adenine substitutions up to and including the -16 position (Figure 4F). However, 
237 substitution of $-14 a$, which marks the start of the SDS, abolished the effect of $25 c>t$ on

238 sfGFP signal suppression, with significantly increased fluorescence in the strain encoding

239 the $14 a>t$ mutation $(p<0.001)$. In order to exclude the possibility that $-14 a>t$ had a non-

240 specific effect on the functionality of the SDS due to enhanced ribosomal binding, we

241 introduced $-14 a>t$ in the WT reporter (without $25 c>t$ ). Similar signal levels were observed

242 in both reporters $(-14 a / 25 c$ and $-14 t / 25 c)$, suggesting that $-14 a>t$ alone does not affect

243 protein expression (Figure 4G).

244 We next investigated the effect of the $-14 a>t$ mutation on OmpK36 abundance in the

245 context of ompK36 ${ }_{\mathrm{WT}}$ and ompK36wT(25c>t). As expected, no effect on abundance was

246 observed when the mutation was introduced into ompK36 ${ }_{W T}$ (Figure $4 \mathrm{H}$ ). In contrast,

247 introduction of the $-14 a>t$ mutation in the 5 'UTR of ompK36 ${ }_{\mathrm{WT}(25 \mathrm{c}>\mathrm{t})}$ increased OmpK36

248 abundance (Figure $4 \mathrm{H}$ ). These findings therefore suggest that the $25 \mathrm{c}>\mathrm{t}$ mutation may

249 result in an inhibitory mRNA secondary structure via a specific interaction with position -

25014 in the 5'UTR.

The 25c>t mutation induces an mRNA stem loop structure involving the Shine-

252 Dalgarno sequence

253 To test the hypothesis that the $25 c>t$ mutation results in an mRNA interaction between -

$25414 \mathrm{a}$ and $25 \mathrm{u}$ that obstructs the SDS and prevents ribosome recruitment, we probed the

255 structures of the ompK36wt and ompK36WT(25c>t) mRNAs using dimethyl sulfate (DMS)

256 mutational profiling with sequencing (DMS-MaPseq) (Zubradt et al., 2017). This technique

257 uses DMS modifications of adenine and cytosine bases in the RNA, detected as 258 mismatches during reverse transcription, to infer the accessibility of individual bases and 259 subsequently build a model of the RNA structure. We found that adenine and cytosines 
within the SDS are more accessible to DMS in ompK36wT mRNA than ompK36WT(25c>t)

261 mRNA both in vitro and in vivo (Figures 5A, 5B, Figure S1). Introducing the 24c>u

262 mutation in addition to $25 c>u$ reversed the DMS accessibility of the SDS to that

264 no DMS treatment, which indeed showed minimal background signal (Figure 5D).

266 Correlation of the DMS signals between different pairs of ompK36 variants (from positions

$267-16$ to +28$)$ further demonstrated higher similarity between ompK36WT and ompK36WT(24\&25c>t) than between ompK36WT and ompK36WT(25c>t) (Figure 5E-G).

269 This implies that the RNA structure of the double mutant more closely resembles the WT.

270 Indeed, DMS-driven structure models showed visually that the SDS is largely accessible

271 for both the WT and the double mutant (although uncertainty exists in the model post 272 position 13 of the double mutant) (Figures $5 \mathrm{H}-\mathrm{J}, \mathrm{SI}$ ). In contrast, the SDS in ompK36WT(25c>t) is sequestered into a stem structure, which incorporates the paired

274 positions of -14 and 25 . These results provide direct evidence that RNA structure 275 occluding the SDS underlies the reduced expression of OmpK36 in strains encoding the $27625 c>t$ mutation. Altogether, these findings provide the precise molecular mechanism 277 linking the occurrence of synonymous SNPs to altered OmpK36 abundance and 278 meropenem resistance levels. 


\section{Discussion}

280 OmpK36 is a key porin in KP, reflected by its typically high abundance in the outer membrane. However, its role in facilitating antibiotic entry has exerted an evolutionary pressure favouring mutations that restrict this process (Vergalli et al., 2020). Using large clinical genome collections, we identified a single, recurrently-emerging synonymous base substitution at the $25^{\text {th }}$ nucleotide position of ompK36 that increases carbapenem resistance by reducing OmpK36 expression. We observed that this $25 c>t$ mutation usually occurs in the absence of L3 insertions, unless a 24c>t mutation which reverses the phenotype is also present, demonstrating that KP typically uses either OmpK36 pore constriction (via L3 insertions) or reduced OmpK36 abundance as a means for increasing resistance. Mechanistically, we show the significant impact a single synonymous substitution can have on protein expression, through the induction of a dramatic mRNA conformational change. To the best of our knowledge, this is the first time an adaptive mutation that restricts translation initiation has been shown to occur naturally.

In unravelling the molecular mechanism, our in vitro mutagenesis experiment showed that reduced OmpK36 expression via the $25 c>t$ mutation is the result of a specific interaction between $25 \mathrm{u}$ in the mRNA and the first adenine base at the upstream SDS (-14a). The interaction was confirmed by solving mRNA structures with DMS-MaPseq. This technique revealed large conformational differences in the structures of the ompK36 ${ }_{\mathrm{WT}}$ and ompK36 ${ }_{\mathrm{WT}(25 \mathrm{c}>\mathrm{t})}$ transcripts, including the presence of a stem loop structure sequestering

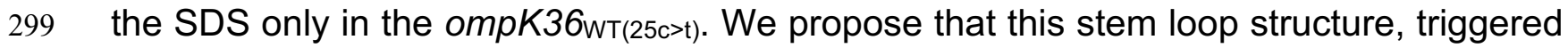
by the interaction between $25 u$ and $-14 a$, impedes ribosomal binding and thereby reduces

301 translation efficiency. Further evidence for this proposed mechanism also came from the 302 mRNA transcript model of ompK36 $\mathrm{WT}_{(24 \& 25 \mathrm{c}>\mathrm{t})}$ (these double mutations also having been 
observed among clinical isolates), which again had a different conformation but showed no stem loop structure obscuring the SDS. This could explain the reversal of the phenotypes observed in this mutant compared with that encoding only $25 c>t$. Indeed, previous studies have demonstrated that experimentally-induced mRNA secondary structures near the SDS decrease protein levels using a synthetic library of synonymous GFP variants (Kudla et al., 2009) and, more recently, using two endogenous genes in $E$. coli (Bhattacharyya et al., 2018). Crucially, however, it has been proposed that the base composition at the start of genes has evolved to minimise the formation of these structures, as evidenced by a genome-wide analysis in E. coli (Bhattacharyya et al., 2018). This appears to be the case in the ompK36 ${ }_{\mathrm{WT}}$ transcript, in which the SDS lies in a largely open conformation. However, the mechanism behind the increased resistance of the $25 c>t$ mutant (and reversal of this phenotype in the $24 \& 25 c>t$ mutant) extends our understanding of this system by demonstrating that mutations which induce alternative secondary structures can also be selected during adaptation. Importantly it also provides a demonstration of the functional use of these inhibitory mRNA structures in regulation among naturally occurring and clinically relevant populations.

In keeping with the key role of OmpK36 in maintaining KP physiology, we demonstrated that the $25 c>t$ mutation decreased bacterial replication in a murine pneumonia model. This correlated with reduced levels of serum IFNy which plays an important role in KP lung infection in mice (Yoshida et al. 2001; Moore et al., 2002). Our phylogenetic analyses suggested that this level of attenuation may be enough to impact on transmission, with most isolates possessing this mutation forming singletons or pairs in the ST258/512 phylogeny (i.e. not being part of larger clonal expansions). Moreover, despite its frequent emergence, the total prevalence of the $25 c>t$ mutation among the ST258/512 genome 
collection remained at $1.2 \%$. This is similar to the prevalence of loss-of-function mutations in ompK36 $(0.7 \%)$ but is in sharp contrast to L3 insertions, which have spread widely via large clonal expansions to reach a prevalence of $47.0 \%$. Nevertheless, despite the limited clonal expansion of $25 \mathrm{c}>\mathrm{t}$, our identification of this mutation in clinical isolates demonstrates that it does not preclude infection in patients.

Using our translational model of pneumonia, we could further show that the increased resistance conferred by the $25 \mathrm{c}>\mathrm{t}$ mutation was enough to result in a clinical impact, thereby explaining its recurrent emergence (despite the fitness cost). Mice infected with $\mathrm{KP}$ expressing ompK36 $\mathrm{wT}_{\mathrm{T}}+\mathrm{KPC}-2$ could be successfully treated with meropenem, while those infected with KP possessing ompK36 ${ }_{\mathrm{WT}(25 \mathrm{c}>\mathrm{t})}+\mathrm{KPC}-2$ could not. We therefore propose that the repeated emergence of $25 \mathrm{c}>\mathrm{t}$ is driven by antibiotics, possibly due to prolonged exposure and/or subtherapeutic dosing; both are commonly encountered among critically ill patients, in whom antibiotic pharmacokinetics are difficult to predict at an individual patient level (Roberts et al., 2014). Indeed, porin modifications occurring within the time course of a single infection have been reported previously (Elliott et al., 2006; Yoshino et al., 2021), demonstrating potential for their emergence and selection given strong antibiotic pressure. Increased host susceptibility due to co-morbidity and impaired immune responses in the population most at risk of KP disease could also render the associated fitness cost of $25 \mathrm{c}>\mathrm{t}$ less significant.

While the frequency of $25 \mathrm{c}>\mathrm{t}$ has remained low, we propose that its ongoing de novo emergence, together with the spectrum of other known OmpK36 modifications, impose a significant impact on patient treatment. Moreover, porin mutations that reduce carbapenem entry are not restricted to $\mathrm{KP}$ and are found in other WHO critical priority organisms such as Pseudomonas aeruginosa and Escherichia coli (Bajaj et al., 2016; 
351 Lister et al., 2009). This highlights the need for the development of novel effective

352 antibiotic therapies that circumvent reliance on diffusion through porins. While this has

353 been achieved via the newly licensed cephalosporin, cefiderocol, which instead enters

354 via siderophore uptake systems (Kohira et al., 2016), additional drugs are still needed.

355 In summary, our combined genomic, experimental and translational approaches have

356 uncovered a novel mechanism underpinning carbapenem resistance, mediated by

357 synonymous mutations that alter ompK36 mRNA secondary structure. The associated

358 dynamics of emergence and expansion of these mutations are at the heart of an

359 evolutionary conflict balancing resistance and virulence requirements in KP. We propose

360 a central role for combining genomic surveillance with in vitro and translational evaluation

361 to further our understanding of resistance mutations, and to design and target our clinical

362 interventions accordingly. 


\section{Acknowledgments}

364 We would like to thank Michelle Yeap for making the vector to delete ompK35, Izabela

365 Glegola-Madjeska for support with animal studies and the Pathogen Informatics group 366 from the Wellcome Sanger Institute for informatics support. We also thank Edward Feil 367 and members of the JPI-AMR-funded SPARK consortium (grant MR/R00241X/1) for 368 access to genomes and metadata used in this study.

\section{$369 \quad$ Funding}

370 This work was funded by a MRC CMBI Studentship award MR/R502376/1 (JLCW), an 371 MRC programme grant (MR/R02671/) and Wellcome Investigator Award (107057/Z/15/Z) 372 (GF), and by the Centre for Genomic Pathogen Surveillance and Li Ka Shing Foundation 373 (SD and DMA).

\section{Author contributions}

JLCW and SD conceived the study. SD performed bioinformatics and genomic analyses.

376 JLCW performed the molecular biology and biochemistry experiments. WWL performed 377 the outer membrane preparations. JLCW, JSG and WWL performed the animal 378 experiments under the supervision of GF. JSG performed cytokine measurements. FM, 379 TG and GMR performed the meropenem resistance assays. JZW performed the DMS380 MaPseq under the supervision of SR. Data analysis was carried out by JLCW, SD and 381 JSG. JLCW, SD and GF wrote the manuscript. All authors reviewed and edited the 382 manuscript (JLCW, SD, JSG, JZW, WWL, FM, TG, GMR, SJB, AC, DMA, SR and GF).

\section{Declaration of Interests}

Authors declare that they have no competing interests. 
bioRxiv preprint doi: https://doi.org/10.1101/2022.01.05.475072; this version posted January 5, 2022. The copyright holder for this preprint (which was not certified by peer review) is the author/funder, who has granted bioRxiv a license to display the preprint in perpetuity. It is mago Figure 1. available under aCC-BY 4.0 International license.

A.

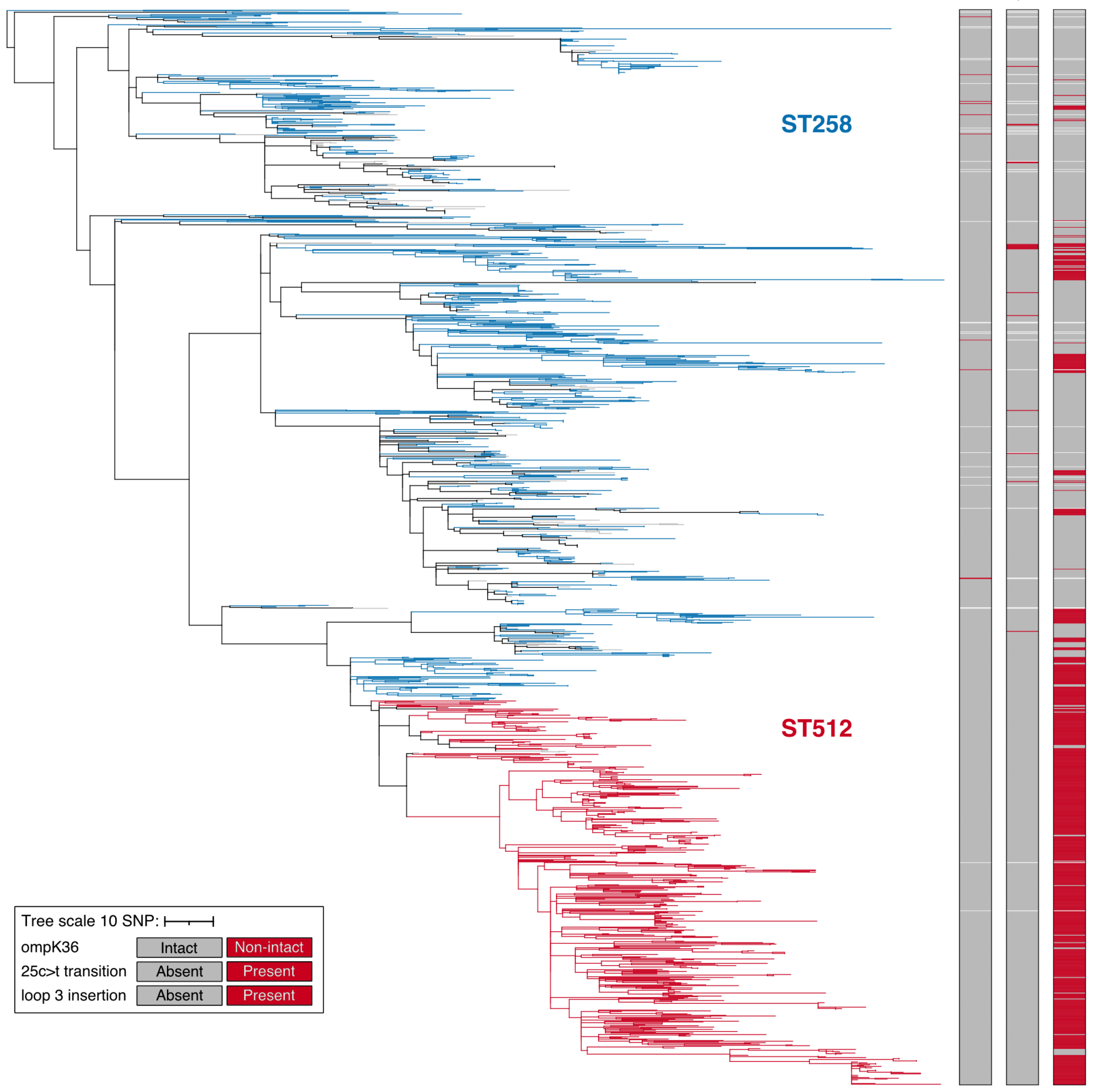

B.

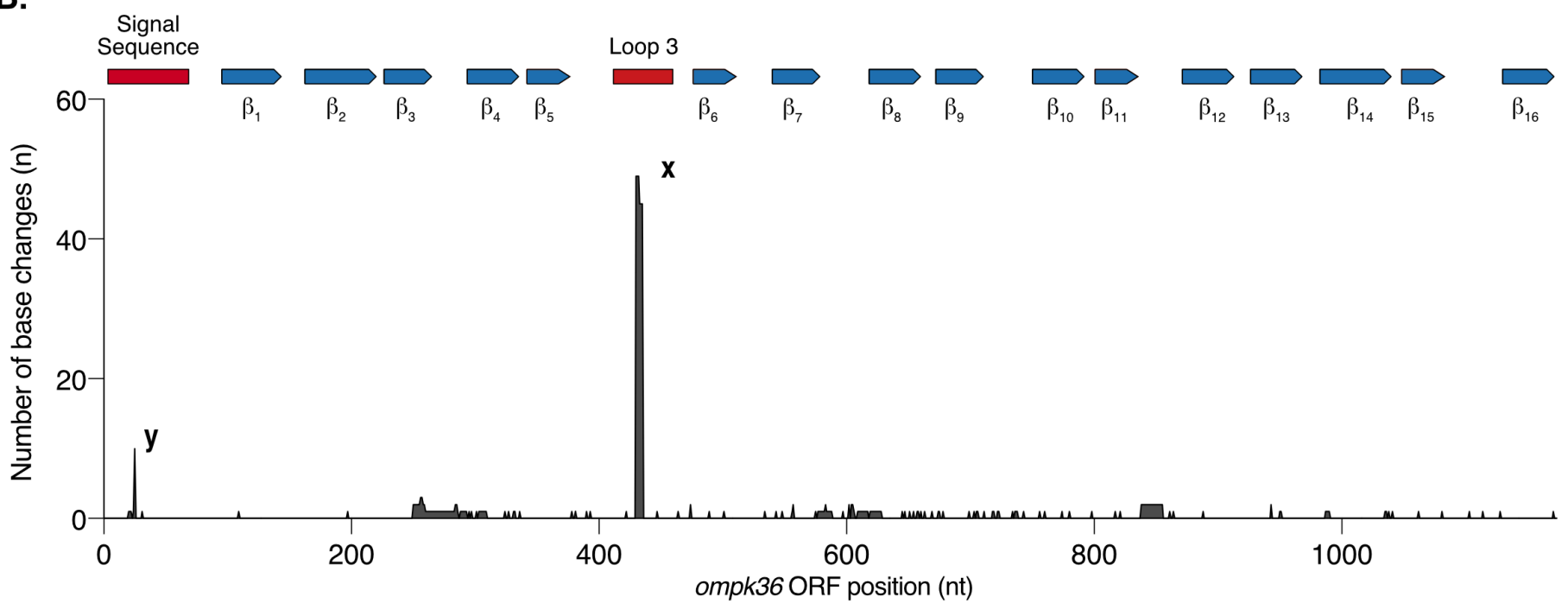



collection of ST258/512 genomes.

A. Phylogenetic tree of 1450 KP ST258/512 isolates constructed using vertically-inherited single nucleotide polymorphisms (SNPs). Branches are coloured blue if all descendent taxa belong to ST258, red if they belong to ST512, grey if they belong to other closely related derivatives of these STs, or black if they belong to one or more of these different categories. Data columns (L-R) show ompK36 (intact/non-intact), ompK36 25c>t transition (present/absent) and ompK36 loop 3 (L3) insertion (present/absent) in each genome. Isolates are marked white if ompK36 could not be unambiguously identified in the genome (columns 1-3) or if ompK36 was non-intact (columns 2/3). The scale represents the number of SNPs per variable site. A similar visualisation is available at: https://microreact.org/project/1vWbaqARPRNc55n4yfdLyQ-ompk36\#wksn-figure-1awong-et-al-2022.

B. The number of changes (including SNPs, insertions and deletions) at each position in ompK36 detected across the ST258/512 tree. The protein structure motifs (signal sequence, beta strands 1-16 and L3) are plotted on top of the sequence as a reference.

402 The labelled peaks represent insertions/reversions within the $L 3(x)$ and the $25 c>t$ 403 mutation found within the signal sequence $(y)$. 
Figure 2. available under aCC-BY 4.0 International license.

A.

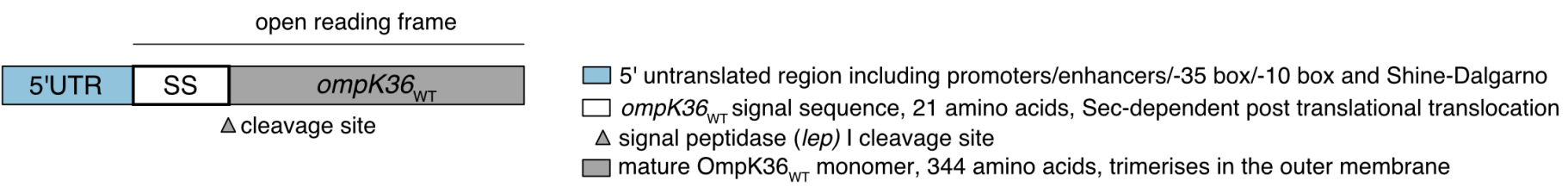

B.

\begin{tabular}{|c|c|c|c|c|c|c|c|c|c|c|c|c|c|c|}
\hline Codon & 1 & 2 & 3 & 4 & 5 & 6 & 7 & 8 & 9 & 10 & 11 & 12 & 13 & 14 \\
\hline ompK36 $6_{\mathrm{wT}}$ & ATG & AAA & GTT & AAA & GTA & CTG & TCC & CTC & CTG & GTA & CCG & GCT & CTG & CTG \\
\hline ompK36 $6_{\mathrm{WT}(25 \mathrm{c}>\mathrm{t})}$ & ATG & AAA & GTT & AAA & GTA & CTG & TCC & CTC & TTG & GTA & CCG & GCT & CTG & CTG \\
\hline ompK36 $6_{\mathrm{WT}(24 \& 25 \mathrm{c}>\mathrm{t})}$ & ATG & AAA & GTT & AAA & GTA & CTG & TCC & CTT & TTG & GTA & CCG & GCT & CTG & CTG \\
\hline Translation & Met & Lys & Val & Lys & Val & Leu & Ser & Leu & Leu & Val & Pro & Ala & Leu & Leu \\
\hline
\end{tabular}

C.

Meropenem MICs in ompK36 variants

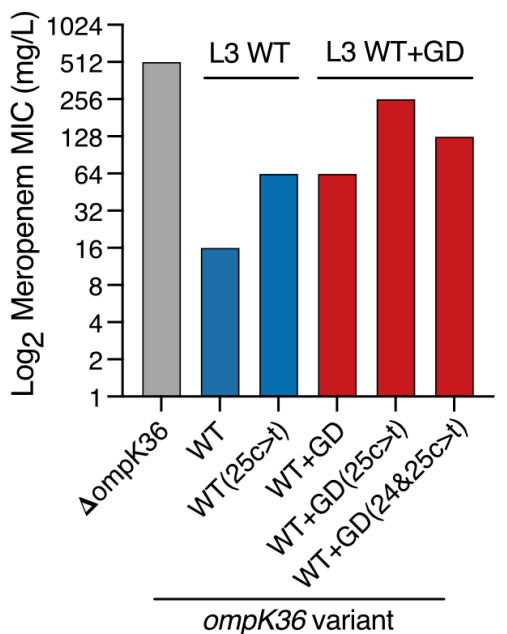

D.

Outer membrane OmpK36 expression in variants

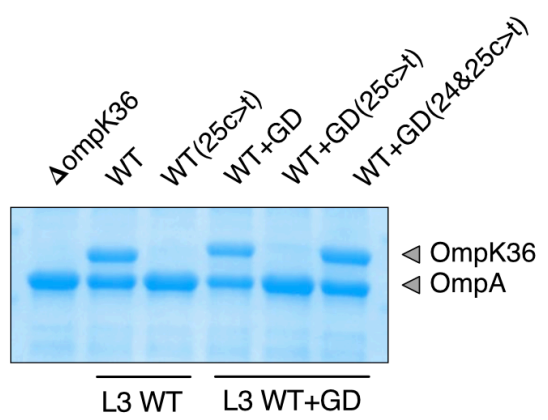

405

406

407

408

409

410

411

Figure. 2. ompK36 ${ }_{\mathrm{WT}(25 \mathrm{c}>\mathrm{t})}$ leads to increased meropenem MIC and reduced OmpK36 abundance.

A. Schematic of the ompK36 locus.

B. The synonymous $25 \mathrm{c}>\mathrm{t}$ mutation occurs at codon position 9 (Leu9) resulting in a change of codon from CTG (ompK36 $\left.{ }_{\mathrm{WT}}\right)$ to TTG (ompK36 $\left.\mathrm{wT}_{\mathrm{W}(25 \mathrm{c}>\mathrm{t})}\right)$. The $24 \mathrm{c}>\mathrm{t}$ mutation occurs in position 8 (Leu8) resulting in a synonymous CTC to CTT codon switch.

C. The $25 c>t$ transition increases the meropenem resistance achieved on both a WT and $\mathrm{WT}+\mathrm{GD}$ ompK36 background. The additional $24 \mathrm{c}>\mathrm{t}(\mathrm{WT}+\mathrm{GD}(24 \& 25 \mathrm{c}>\mathrm{t}))$ mutation partially reverses the resistance achieved in the $25 c>t$ transition. 
415 All strains harbour a pKpQIL-like plasmid expressing KPC-2 and have ompK35 deleted.

416 Resistance values represent broth MICs (median of 3 repeats).

417 D. Representative Coomassie gel image of outer membrane (OM) preparations

418 demonstrating the reduced OmpK36 expression conferred by $25 c>t$ in both WT and

$419 W T+G D$ backgrounds. The additional $24 c>t$ in $W T+G D(24 \& 25 c>t)$ reverses the OM

420 depletion of OmpK36 in WT+GD(25c>t). 
bioRxiv preprint dol: https://doi.org/10.1101/2022.01.05.475072; this version posted January 5, 2022. The copyright holder for this reprint (which was not certified by peer review) is the author/funder, who has granted bioRxiv a license to display the preprint in perpetuity. It is made Figure 3.

A.

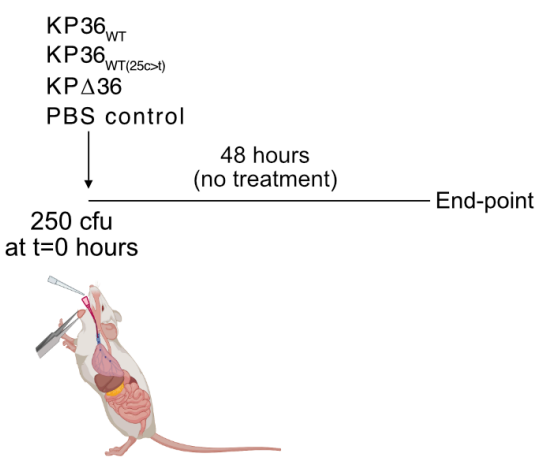

D. CFU Blood

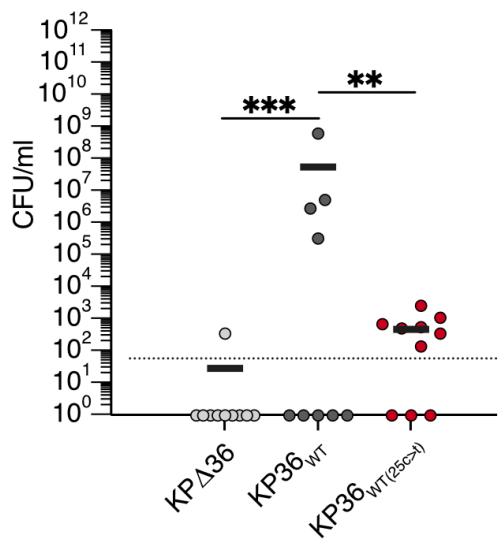

F.

Meropenem $100 \mathrm{mg} / \mathrm{kg}$ hourly $\mathrm{x} 4$

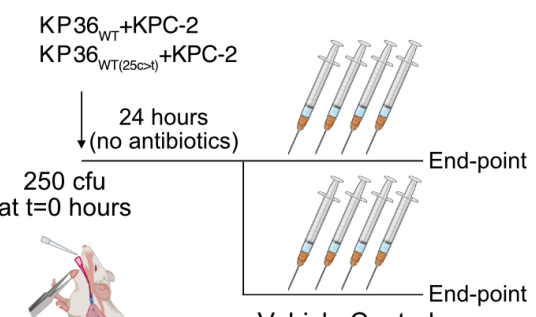

Vehicle Control

I.

CFU Blood

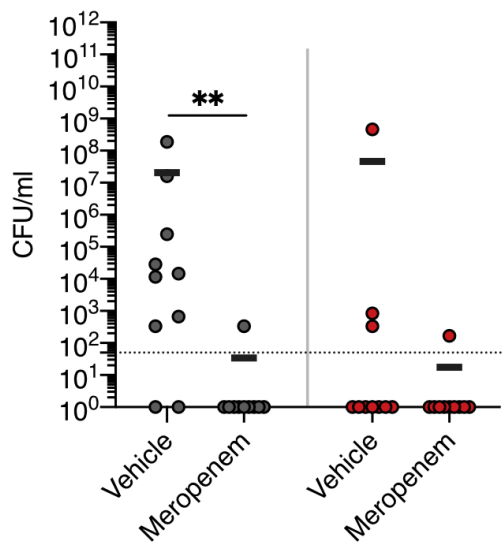

B.

Weight Loss
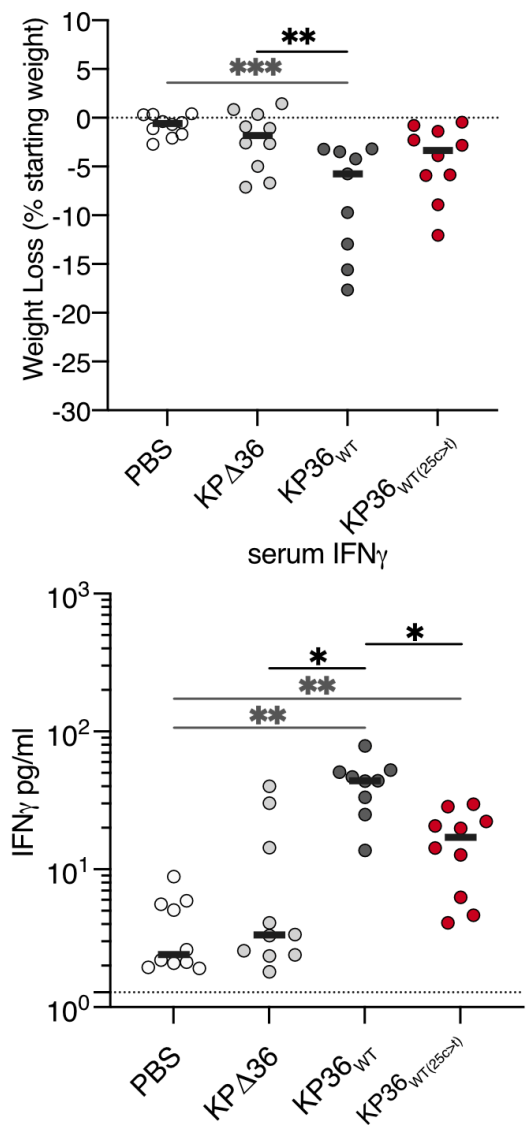

G.

Weight Loss

H.

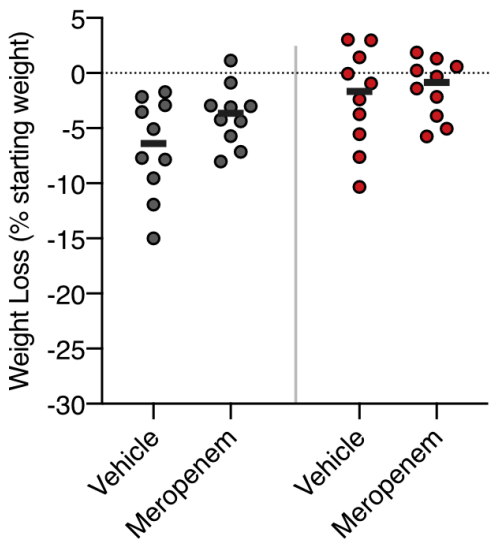

J.

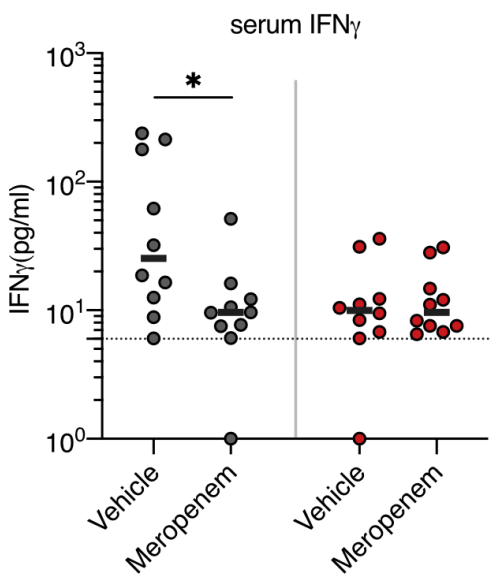

C.

CFU Lungs
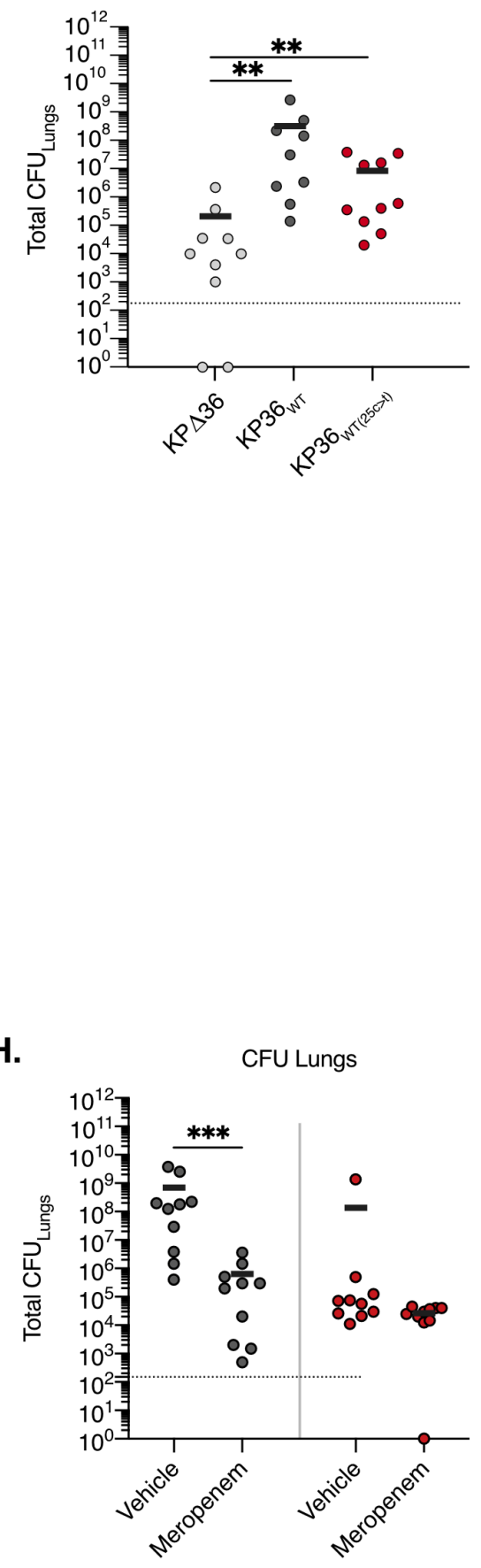

context of meropenem therapy.

A-E. Mice were infected by intubation and administration of 250 CFU of KP36WT, KP36 ${ }_{\mathrm{WT}(25 \mathrm{c}>\mathrm{t})}$ or KP $\Delta 36$ strains; mock infection with PBS was used as a control. A schematic of the infection protocol is outlined in panel a.

B. Animals infected with KP36wт show greater weight loss after $48 \mathrm{~h}$ compared to mockinfected animals and those infected with KP $\Delta 36$. Weight loss in animals infected with $\mathrm{KP} 36_{\mathrm{WT}(25 \mathrm{c}>\mathrm{t})}$ is not significantly different to that of the PBS or KP $\Delta 36$ groups. that infection with KP $\Delta 36$ does not result in high lung bacterial burdens and bacteraemia. Infection with KP36wT results in high pulmonary bacterial burdens (C) and bacteraemia (D), while infection with KP36 ${ }_{W T(25 c>t)}$ results in a trend towards lower pulmonary bacterial counts and reduced levels of bacteria in the blood. Dotted line represents the limit of detection of the assay.

E. Infection with $\mathrm{KP} 36_{\mathrm{WT}}$ or $\mathrm{KP} 6_{\mathrm{WT}(25 \mathrm{c}>\mathrm{t})}$ leads to increased serum IFNy compared to mock-infected (PBS) animals. KP36 ${ }_{\mathrm{WT}(25 \mathrm{c}>\mathrm{t})}$ infection results in significantly reduced serum IFNy levels compared to KP36wt infection.

F-J. Mice were administered with KP36 $w_{T}+K P C-2$ or KP36 ${ }_{W T(25 c>t)}+K P C-2$ strains and subjected to a meropenem dosing regimen to assess the success of antibiotic therapy in KP-induced severe pneumonia. A schematic of the infection protocol is outlined in panel F.

G. No changes were observed in weight loss between meropenem and vehicle control 445 therapy when experimental pneumonia induced by either $\mathrm{KP} 36{ }_{\mathrm{WT}}+\mathrm{KPC}-2$ or 
$447 \mathbf{H}$ and I. CFU enumeration in lungs $(\mathbf{H})$ and blood (I) shows significant reduction in 448 meropenem-treated animals infected with KP36 ${ }_{W T}+\mathrm{KPC}-2$ compared to vehicle treated 449 animals; levels of bacteraemia were undetectable in all but one meropenem treated 450 animal. Meropenem treatment has no impact on lung or blood CFUs in animals infected 451 with KP36 $\mathrm{WT}_{(25 \mathrm{c}>\mathrm{t})}+\mathrm{KPC}-2$.

452 J. Serum levels of IFNy are significantly reduced after meropenem treatment in animals 453 infected with KP36 ${ }_{W T}+\mathrm{KPC}-2$; cytokine levels are not affected by meropenem treatment 454 in animals infected with KP36 ${ }_{\mathrm{WT}(25 \mathrm{c}>\mathrm{t})}+\mathrm{KPC}-2$.

455 Graphs show median values of 2 biological repeats (4-5 mice per group). Statistical 456 significance was determined by one-way ANOVA with Tukeys's multiple comparison 457 post-test. ${ }^{*}, \mathrm{P}<0.05 ;{ }^{* *}, \mathrm{P}<0.01 ;{ }^{* *} \mathrm{P}<0.001 ;{ }^{* * *}, \mathrm{P}<0.0001$ in $\mathrm{B}-\mathrm{E}$. The statistical 458 significance of the comparisons between vehicle and meropenem-treated animals was 459 determined via a non-parametric Mann-Whitney test. ${ }^{*}, \mathrm{P}<0.05 ;{ }^{* *}, \mathrm{P}<0.01 ;{ }^{* * *} \mathrm{P}<0.001$ 460 in G-J. 


\section{Figure 4.}

A.

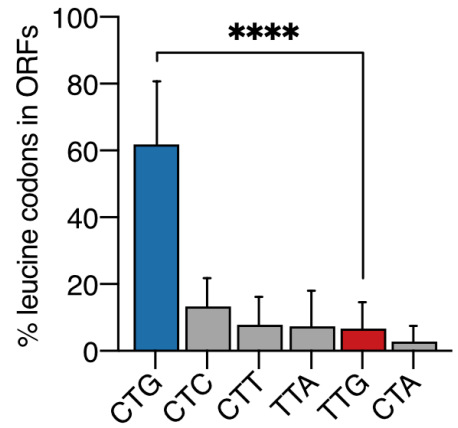

B.

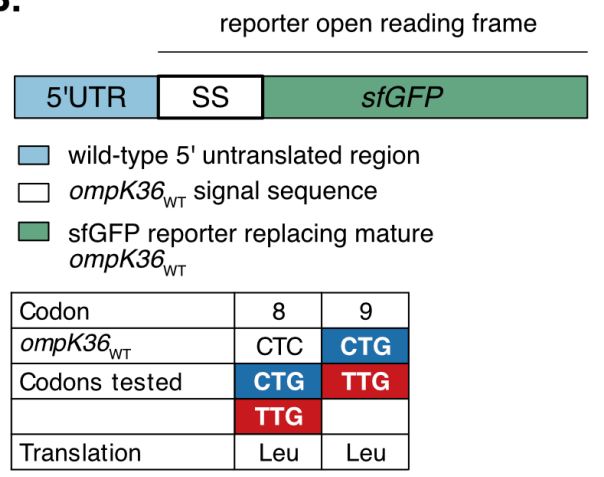

C.

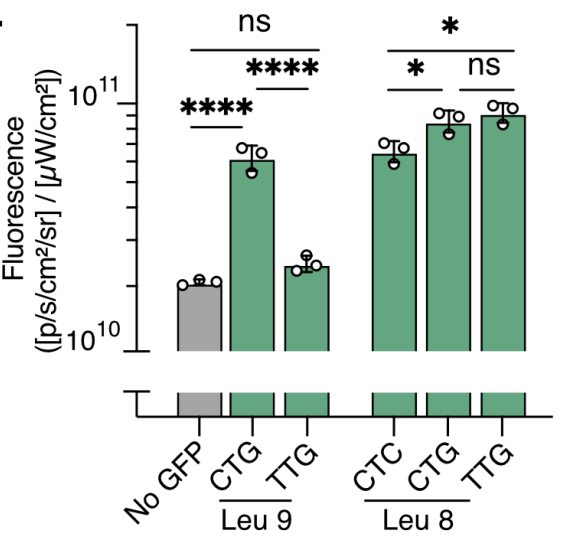

D. -10 box Shine-Dalgarno Sequence ompK36 start codon 5 ' - c a g tggcat a a ta a a g g a ta ta a c a a a c a agg t t a a t a c a tg ATG- 3 ' $-32$ (transcription start site) $-14$

$\mathbf{F}$.

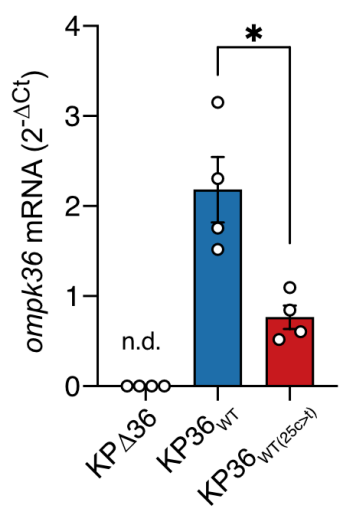

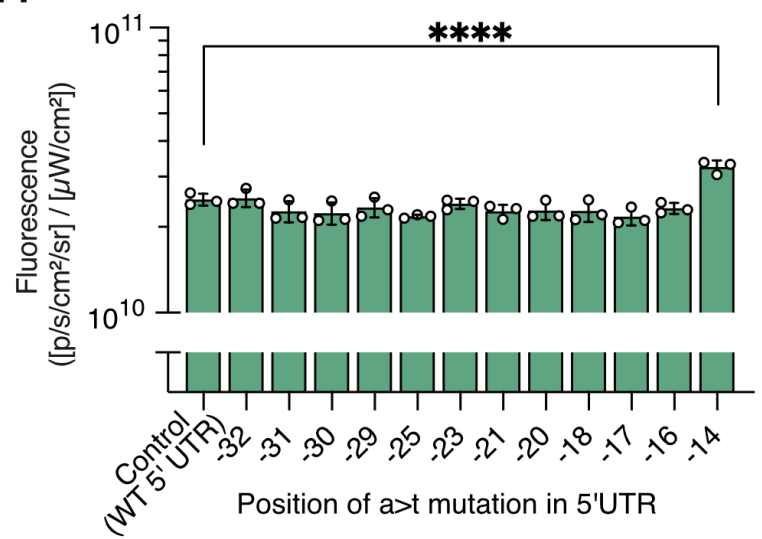

G.

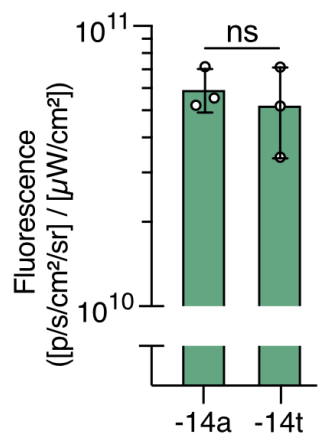

H. $\begin{array}{llllllll}\mathrm{L} & 1 & 2 & 3 & \mathrm{~L} & 4 & 5 & 6\end{array}$

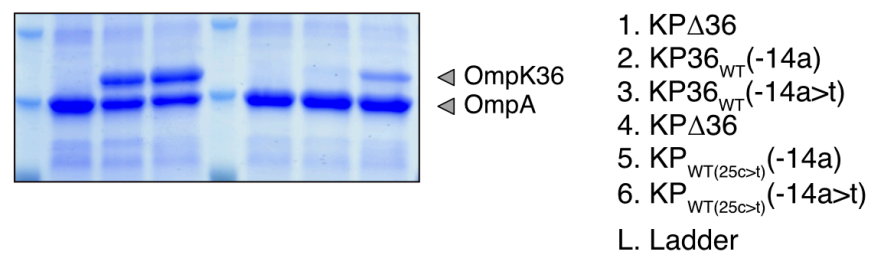

461

462 Figure 4. The $25 \mathrm{c}>\mathrm{t}$ mutation results in a specific intramolecular interaction

463 between $-14 a$ and $25 u$ in the mRNA transcript.

464 A. Proportion of leucine residues encoded by each of the six codons across individual 465 open reading frames (ORFs) in the ICC8001 KP genome. A significant difference was 
observed in the use of CTG relative to TTG; significance was determined by paired T-test $* * * *, p<0.0001$

B. Schematic of the sfGFP reporter. The sequence encoding the mature OmpK36 protein was replaced with sfGFP, generating a chimeric fusion between the ompK36 ${ }_{\mathrm{WT}}$ signal sequence (Leu9 CTG) and sfGFP. The inset table describes the mutations that were introduced.

C. Mutation of Leu9 CTG (ompK36wT) to TTG (ompK36 $\mathrm{WT}(25 \mathrm{c}>\mathrm{t})$ ) decreases expression of the sfGFP reporter. Mutations in Leu8 (CTC codon in ompK36 ${ }_{\mathrm{WT}}$ ) to CTG or TTG codons lead to similarly increased levels of reporter expression. Fluorescence was determined using IVIS SpectrumCT. Graphs show means of 3 biological repeats \pm the standard deviation (SD). Statistical significance was determined by one-way ANOVA with Tukey's multiple comparison test. ns, not significant; ${ }^{*}, \mathrm{P}<0.05 ;{ }^{* * * *}, \mathrm{P}<0.0001$.

D. Sequence of the ompK36 5'UTR. Adenine bases in the 5'UTR (in bold) were individually mutated to thymine.

E. RT-qPCR analysis of ompK36 shows decreased transcript levels in the strain encoding ompK36 ${ }_{\mathrm{WT}(25 \mathrm{c}>\mathrm{t})}$ compared to ompK36 $\mathrm{WT}$. Bar charts show the mean \pm the standard error of the mean (SEM) of 4 biological repeats. nd, not detected. ${ }^{* *} \mathrm{P}<0.005$ by paired Student's $t$ test.

F. Expression of the sfGFP reporter encoding the ompK36 ${ }_{W T(25 c>t)}$ signal sequence is specifically increased upon mutation of the adenine in position -14 to thymine $(-14 a>t)$. The $-14 a$ marks the start of the Shine-Dalgarno sequence (SDS). Fluorescence was determined using IVIS SpectrumCT. Graphs show the means \pm SD of 3 biological repeats. Statistical analysis was performed by one-way ANOVA with Dunnett's multiple comparison post-test. $^{* * * *}, \mathrm{P}<0.0001$. 
490 G. Mutation of the adenine in position -14 to thymine $(-14 a>t)$ in the reporter construct

491 encoding the ompK36wт signal sequence (Figure 4B) does not impact sfGFP expression.

492 Bar charts show the mean \pm the standard deviation (SD) of 3 biological repeats. ns, not

493 significant by paired Student's $t$ test.

494 H. Representative Coomassie gel image of outer membrane preparations from isogenic

$495 \mathrm{KP}$ strains with the indicated ompK36 mutations in positions -14 (5'UTR) and 25 (ORF

496 signal sequence). Mutation of the adenine in position -14 to thymine $(-14 a>t)$ restores

497 expression of OmpK36 in the presence of the $25 c>t$ mutation. 
bioRxiv preprint doi: https://doi.org/10.1101/2022.01.05.475072; this version posted January 5, 2022. The copyright holder for this preprint (which was not certified by peer review) is the author/funder, who has granted bioRxiv a license to display the preprint in perpetuity. It is made available under aCC-BY 4.0 International license.

\section{Figure 5.}

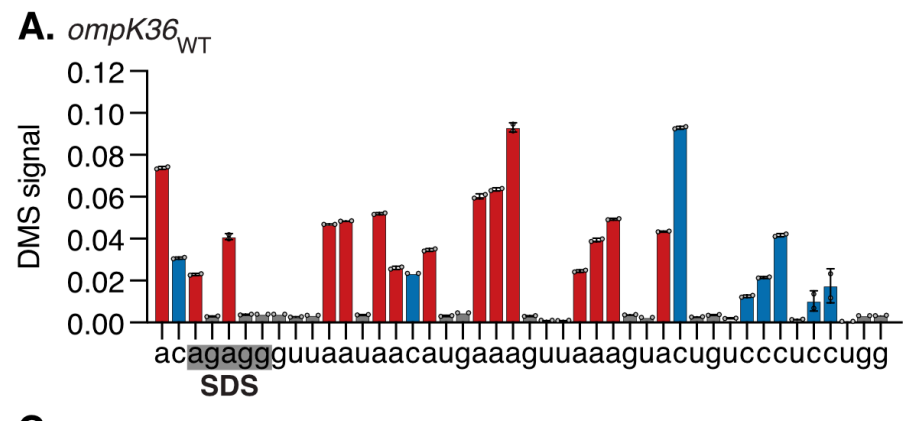

C. $o m p K 36_{\mathrm{WT}(24 \& 25 c>t)}$

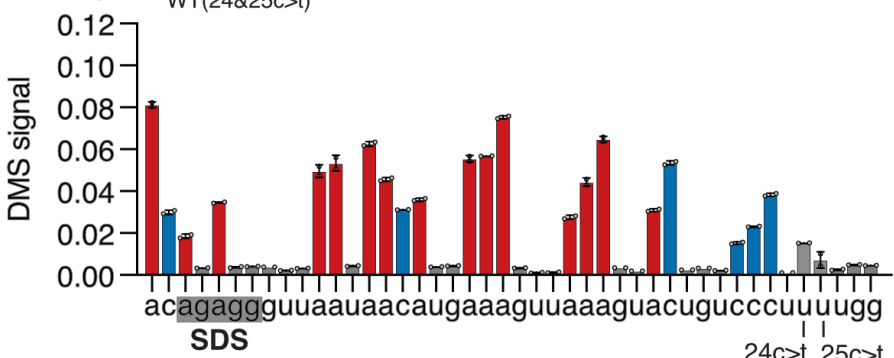

B. $o m p K 36_{\mathrm{WT}(25 c>t)}$

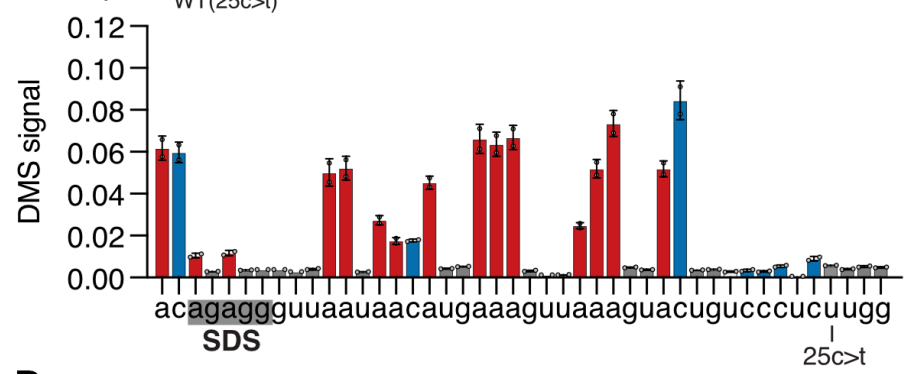

D. ompK36 ${ }_{\mathrm{WT}}$ no DMS

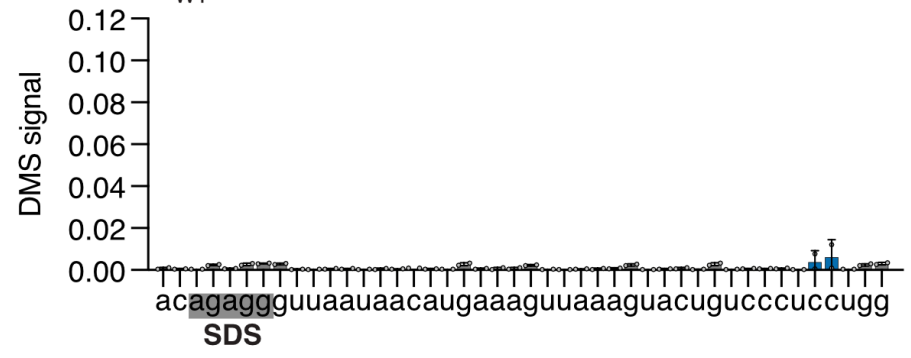

E. $o m p K 36_{W T}$ vs. $o m p K 36_{W T(25 c>t)}$

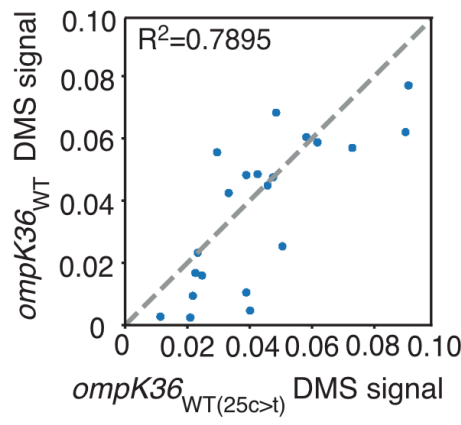

F. $о m p K 36_{\mathrm{WT}}$ vs. $о \mathrm{mp} K 36_{\mathrm{WT}(24 \& 25 c>t)}$

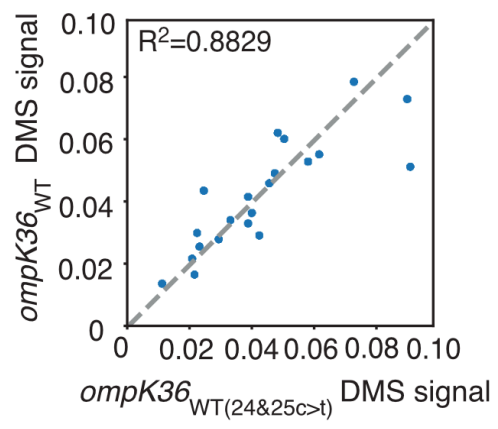

G. ompK36 ${ }_{\mathrm{WT}}$ replicates

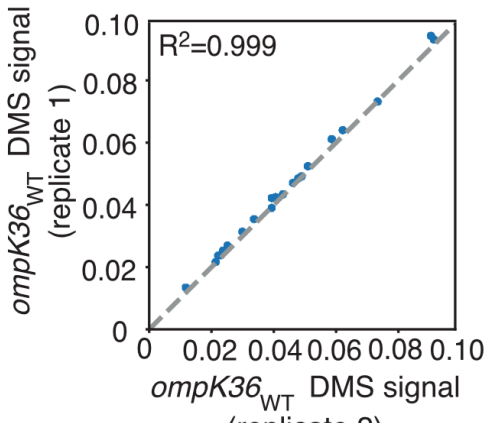

(replicate 2)

H. $о m p K 36_{\mathrm{WT}}$

I. $\mathrm{ompK} 36_{\mathrm{WT}(25 \mathrm{c}>t)}$

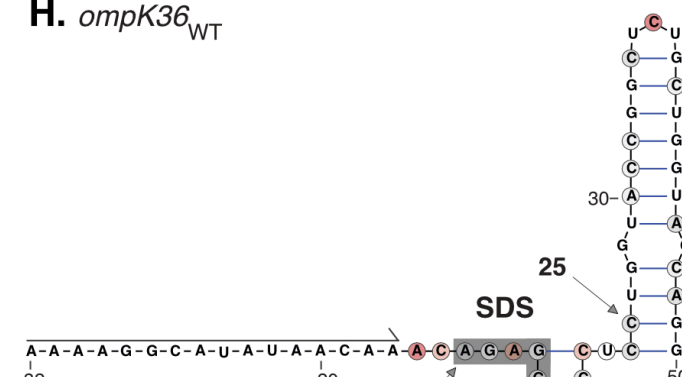

$-32$

$-20$

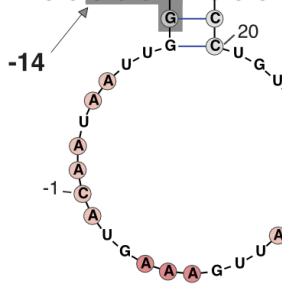

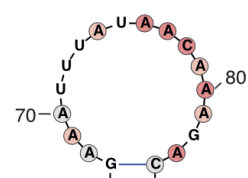

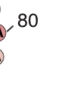

J. ompK36 ${ }_{\mathrm{WT}(24 \& 25 c>t)}$

SDS

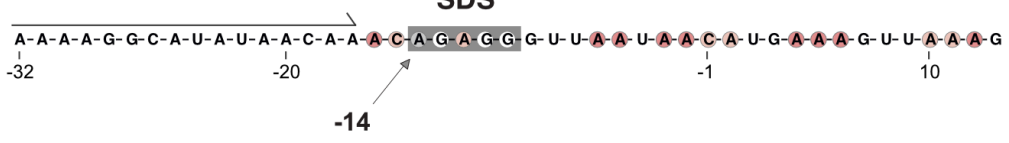

normalised DMS signal $\begin{array}{lllll}0.0 & 0.3 & 0.6 & 1.0\end{array}$ 

Dalgarno sequence (SDS).

A-D Dimethyl sulfate (DMS) signal per nucleotide from full length in vitro-transcribed and

502 refolded ompK36, where higher values correspond to increased base accessibility. DMS 503 signal and error bars for $\mathrm{nt}-16 \mathrm{a}$ through $28 \mathrm{~g}$ are shown for ompK36WT

504 (A), ompK36 ${ }_{\mathrm{WT}(25 \mathrm{c}>\mathrm{t})}(\mathbf{B})$, ompk36wT(24\&25c>t) $(\mathbf{C})$ and DMS-untreated ompK36wT (D).

E-G. Comparison of DMS reactivities from nucleotides $-16 \mathrm{a}$ through $28 \mathrm{~g}$ between ompK36 $6_{\mathrm{WT}}$ and ompK36 $\mathrm{WWT}_{\mathrm{W}(25 \mathrm{c}>\mathrm{t})}(\mathbf{E})$, between ompK36 $6_{\mathrm{WT}}$ and ompk36 $6_{\mathrm{WT}(24 \& 25 \mathrm{c}>\mathrm{t})}(\mathbf{F})$, and

507 between ompK36 ${ }_{\mathrm{WT}}$ replicates $(\mathbf{G})$. Pearson correlations $\left(\mathrm{R}^{2}\right)$ are shown.

508 H-J. DMS-constrained structure models of the 5' end of ompK36 ${ }_{\mathrm{WT}}(\mathbf{H})$, ompK36 $6_{\mathrm{WT}(25 \mathrm{c}>\mathrm{t})}$

509 (I) and ompK36 $\mathrm{WT}(24 \& 25 \mathrm{c}>\mathrm{t})(\mathbf{J})$. Nucleotides are colored by normalized DMS signal. The 510 PCR primer binding sites where DMS information is unavailable are marked from 511 positions -32 to -17 . The SDS in RNA structures are highlighted in grey. Arrows indicate 512 the -14 and +25 positions.

\section{Supplementary Figure Legends}

515 Figure S1. Position 25 in the ompK36 ${ }_{\mathrm{WT}(25 \mathrm{c}>\mathrm{t})}$ RNA forms a stem with the Shine516 Dalgarno sequence (SDS) in vivo.

517 A. Dimethyl sulfate (DMS)-constrained predicted structures of in vivo ompK36wT (top) and

518 ompK36 ${ }_{\mathrm{WT}(25 \mathrm{c}>\mathrm{t})}$ (bottom) RNA molecules. Nucleotides are colored by normalized DMS519 MaPseq mutation rate (DMS signal), where higher values correspond to increased base 520 accessibility. The PCR primer binding site where DMS information is unavailable is 521 highlighted. In vivo RNA structures correspond with in vitro transcribed RNA structures 
522 (Figure 5A\&B) with SDS sequestration in the ompK36 $\mathrm{WT}_{\mathrm{W}(25 \mathrm{c}>\mathrm{t})}$ but not ompK36 $\mathrm{WT}$ RNA

523 molecule.

524 B. Correlation of DMS signals for each nucleotide between two biological replicates for

525 in vivo ompK36 ${ }_{\mathrm{WT}}$ (top) and ompK36 ${ }_{\mathrm{WT}(25 \mathrm{c}>\mathrm{t})}$ (bottom).

526 C. DMS signal per nucleotide of in vivo ompK36 $\mathrm{WT}_{\mathrm{T}}$ (top) and ompK36 $\mathrm{WT}(25 \mathrm{c}>\mathrm{t})$ (middle) and

527 DMS-untreated ompK36wt (bottom) RNA molecules. The SDS are highlighted in grey 528 boxes. 


\section{Tables}

530 Table 1. ompK36 variants and KP strains used in this study.

\begin{tabular}{|c|c|c|c|}
\hline ompK36 variants & Nucleotide at & Nucleotide at & Loop 3 (L3) insertion \\
\hline ompK36 & c & c & Absent \\
\hline ompK36 $\mathrm{WT}(25 \mathrm{c}>\mathrm{t})$ & c & $\mathrm{t}$ & Absent \\
\hline ompK36 ${ }_{W T+G D}$ & c & c & Glycine-Aspartate (GD) \\
\hline ompK36 ${ }_{W T+G D(25 c>t)}$ & c & $\mathrm{t}$ & Glycine-Aspartate (GD) \\
\hline ompK36 ${ }_{\mathrm{WT}+\mathrm{GD}(24 \& 25 \mathrm{c}>\mathrm{t})}$ & $\mathrm{t}$ & $\mathrm{t}$ & Glycine-Aspartate (GD) \\
\hline Strains & ompK35 & ompK36 & Carbapenemase \\
\hline $\mathrm{KP} \Delta 36$ & $\Delta$ & $\Delta$ & $+/-\mathrm{KPC}-2^{*}$ \\
\hline KP36 ${ }_{W T}(+/-K P C-2)$ & $\Delta$ & ompK36 & $+/-\mathrm{KPC}-2^{*}$ \\
\hline $\mathrm{KP} 36_{\mathrm{WT}(25 \mathrm{c}>\mathrm{t})}(+/-\mathrm{KPC}-2)$ & $\Delta$ & ompK36 ${ }_{\mathrm{WT}(25 \mathrm{c}>\mathrm{t})}$ & $+/-\mathrm{KPC}-2^{*}$ \\
\hline
\end{tabular}

${ }^{*} \mathrm{KPC}-2$ on pKpQIL-like plasmid

534 Table S1. Metadata and genotyping data for a curated collection of 1450 KP ST258/512 535 genomes.

536 Table S2. Metadata and genotyping data for 16,086 KP genomes available in 537 Pathogenwatch. 


\section{Methods}

Genome collections

540 We used two genome collections to characterise the diversity and phylogenetic 541 distribution of ompK36 (and ompK35) variants. The first comprises 1450 public KP 542 genomes belonging to STs 258, 512 and other nested STs, together with curated 543 metadata obtained from associated publications (Table S1). We obtained raw sequence 544 reads if available (1340 isolates), and short-read assemblies from the remainder (110 isolates). Assemblies were generated for all isolates with available raw sequence data using SPAdes v3.9.0 (Bankevich et al., 2012), and annotated with Prokka v1.14.5

547 (Seemann, 2014). Kleborate v1.0.0 (Lam et al., 2021) was used for confirming the ST of 548 each genome and determining the resistance gene content.

549 The second collection comprises a public genome collection of $16,086 \mathrm{KP}$ available in 550 Pathogenwatch together with available metadata and genotyping data (Argimon et al., 2021) (https://pathogen.watch/genomes/all?genusld=570\&speciesld=573) (Table S2).

Phylogenetic analysis of the ST258/512 collection

554 To generate a phylogenetic tree of the ST258/512 collection, we first used the 555 "to_perfect_reads" function within Fastaq v3.17.0 (https://github.com/sangerpathogens/Fastaq) to generate pseudo sequence reads for isolates where only an assembly was available. We then mapped all sequence reads to the reference genome, NJST258_1 (accession CP006923) (Deleo et al., 2014), using Burrows Wheeler Aligner v0.7.17 (Li \& Durbin, 2009). A pipeline comprising SAMtools mpileup v0.1.19 (Li et al., 560 2009) and BCFtools v0.1.19 was used to call SNPs and generate a pseudo-genome 561 alignment. Gubbins v2.4.1 (Croucher et al., 2015) was used to remove recombined 
562 regions from the alignment and generate a maximum likelihood tree with the remaining

563 variable positions. The phylogenetic tree was rooted using an outgroup isolate from a

564 closely-related ST, ST895 (accession SRR5385992), which was later removed from the

565 tree.

$566 \quad$ Identification and characterisation of ompK35 and ompK36 genes

567 The ompK35 and ompK36 genes were identified in all short-read assemblies by

568 performing BLASTn v2.6.0 (Altschul et al., 1990) with a query gene from the reference

569 genome, ATCC43816 (the parental strain of ICC8001 (Wong et al., 2019)) (accession

570 CP009208). We required a single hit of each gene per genome that matched $\geq 10 \%$ of the

571 query length, possessed $\geq 90 \%$ nucleotide similarity and contained a start codon in order

572 to unambiguously identify the gene. Nucleotide sequences were translated to protein sequences in Seaview v4.7 (Gouy et al., 2010) using the standard genetic code, and the protein lengths were determined. Protein sequences were predicted to be intact if they contained a signal sequence, a L3 sequence and sixteen beta-barrel sequences, as determined using BLASTx v2.6.0 (Gish \& States, 1993). Intact protein sequences of each porin were aligned using MUSCLE v3.8 (Edgar, 2004) and the different variants present were identified, taking into account all amino acid substitutions, insertions and deletions. Analysis of ompK36 variation curated manually to ensure accurate positioning of bases around the L3 region (some of which were misaligned due to indels). We then used the sequences from the curated

583 alignment to infer the likely base harboured by each internal node of the ST258/512 584 phylogenetic tree at each position in ompK36. This ancestral reconstruction was 
585

586

587

588

589

590

591

592

593

594

595

596

597

598

599

600

601

602

603

604

605

606

607

608

performed with PastML v1.9.30 (Ishikawa et al., 2019) using maximum parsimony with the accelerated transformation (ACCTRAN) model. Using the predicted states of all internal nodes and the known bases in all terminal nodes, we determined the total number of changes at each position that had occurred across the ST258/512 tree.

Codon usage

The frequency of different leucine codons was determined across all protein-coding genes in the ATCC43816 reference genome, as well as all annotated assemblies in the ST258/512 collection, using the EMBOSS v6.6.0.0 "cusp" tool (http://emboss.sourceforge.net/apps/release/6.3/emboss/apps/cusp.html).

$\underline{5}$ ' untranslated region analysis

The 500bp upstream region of ompK36 was analysed and annotated in Fig. 4D using BPROM (Solovyez \& Salamov, 2011). The SDS was identified manually by consensus with other KP genes.

\section{General cloning, molecular biology and strain generation}

All in vitro assays and animal infections with KP were carried out using ICC8001, a strain derived by animal passage from ATCC43816 (Wong et al., 2019).

A homologous recombination technique resulting in scarless markerless mutants was used to generate all strains based on the Standard European Vector Architecture platform pSEVA612S (JX560380.2) mutagenesis plasmid. Briefly, mutants were generated in two sequential recombination steps. The first step integrates the mutagenesis plasmid into the genome generating a merodiploid. The mutagenic plasmid was introduced by three part conjugation and homologous recombination promoted by lambda-recombinase expressed from a helper plasmid. The second step induces double strand DNA breaks by induction (L-arabinose) of I-Scel expression from the helper plasmid. Double stranded 
609 breaks are induced at I-Scel sites located on the chromosomally integrated mutagenesis

610 vector, flanking the final integration region. This technique has been used to efficiently

611 recombineer in KP (Wong et al., 2019) and other Enterobacteriaceae (Berger et al., 2017)

612 as described in respective manuscripts.

613 Mutagenesis vectors were generated by routine molecular biological techniques. These

614 included plasmid preparation (Monarch Plasmid Miniprep Kit, NEB), genomic DNA 615 extraction from bacterial cells (DNEasy, QIAGEN), polymerase chain reaction (Q5 High-

616 Fidelity 2x Master Mix and OneTaq, Quick-Load 2X Master Mix, both NEB), PCR clean-

617 up (Monarch PCR and DNA Cleanup Kit, NEB) and gel extraction (Monarch Gel

618 Extraction Kit, NEB), site directed mutagenesis (KLD enzyme blend, NEB) and Gibson

619 assembly (Gibson Assembly Master Mix, NEB). Sanger sequencing was carried out to

620 check vector construction and seamless integration into the genome (Eurofins

621 Genomics). Custom primers in this study were made by Sigma-Aldrich. ompK35 mutagenesis

ompK35 wild-type ORF (+500bp flanking regions) was cloned from ICC8001 genomic

624 DNA with primers (P) 1/P2 and ligated by Gibson Assembly into pSEVA612S, linearised with P3/P4. The ORF was removed by inverse PCR to generate the genomic deletion 626 vector (P5/P6) and plasmid recircularized from PCR product (KLD enzyme blend, NEB). ompK36 mutagenesis

ICC8001هompK36 was generated in previous work (Vector 5 in Wong et al., 2019). ompK36 wild-type ORF (+500bp flanking regions) was cloned from ICC8001 genomic DNA with primers P7/P8 and ligated by Gibson assembly into pSEVA612S, linearised 631 with P3/P4. In screening colonies by Sanger Sequencing we found the $25 c>t$ mutation 632 and this was not generated by site directed mutagenesis. 


\section{$\underline{\text { sfGFP vectors and }-14 a>t \text { vectors }}$}

Leucine 9 CTG-sfGFP (wild-type) and Leucine 9 TTG-sfGFP (25c>t transition). The wildtype and 25c>t Sec signal sequences were cloned from the ompK36 vectors generated above with P9/P10. sfGFP was amplified from our previously published glmS site insertion vector (Vector 13 in Wong et al., 2019) with P11/P12 and chimeric fusion generated in the mutagenesis vector ligated by Gibson Assembly.

Leucine 8 CTG-sfGFP and Leucine 8 TTG-sfGFP were generated by site-directed mutagenesis using P13/P14 and P15/P16 respectively using the Leucine 9 CTG-sfGFP (wild-type) as the template.

5' untranslated region mutagenesis of Leucine 9 TTG-sfGFP (25c>t transition). Mutants were generated by site-directed mutagenesis as follows: $-32(a>t)(P 17 / P 18),-31(a>t)$ (P17/P19), $\quad-30(a>t) \quad(P 17 / P 20), \quad-29(a>t) \quad(P 17 / P 21), \quad-25(a>t) \quad(P 22 / P 23), \quad-23(a>t)$
(P22/P24), -21(a>t)
(P22/P25), $\quad-20(a>t)$
(P22/P26), -18(a>t) (P22/P27), -17(a>t)

(P22/P28), -16(a>t) (P22/P29), -14 a>t(a>t) (P22/P30) using leucine 9 TTG sfGFP as the template. The same primer pair (P22/P30) was used to introduce the $-14 a>t$ mutation into Leucine 8 CTG-sfGFP, ompK36WT and ompK36WT(25c>t) vectors.

\section{Introduction of pKpQIL KPC-2 plasmid}

We introduced pKpQIL KPC-2 by conjugation using an E. coli donor following our previously published protocol (Wong et al., 2019).

\section{Meropenem Minimum Inhibitory Concentrations (MIC)}

The meropenem MIC of strains was assessed by broth microdilution by broth microdilution in biological triplicate according to the ISO 20776-1:2019 standard and the median value taken as the final MIC.

\section{$\underline{\text { Outer membrane preparations and gel electrophoresis }}$}


Outer membrane proteins were purified according to a previously described protocol with several modifications (Wise et al., 2018). Saturated overnight cultures of bacteria were grown in LB $(10 \mathrm{~g} / \mathrm{L} \mathrm{NaCl})$ and sonification was performed at $25 \%$ amplitude for 10 bursts

660 of 15 seconds each. $10 \mu \mathrm{g}$ of protein was separated by SDS-PAGE using $12 \%$ acrylamide

661 Mini-protean TGX precast gels (Bio-Rad, USA). Gels were stained with Coomassie 662 (Sigma-Aldrich) and imaged on a ChemiDoc XRS+ (Biorad, USA).

663 Animal work

8-10 week old, female, 18-20g BALB/c mice were purchased from Charles River, UK. All 665 animal work took place in the institution animal facility (Association for Assessment and 666 Accreditation of Laboratory Animal Care accredited) under the auspices of the Animals 667 (Scientific Procedures) Act 1986 (PP7392693). Work was approved locally by the institutional ethics committee.

669 Housing and Randomisation

670 Upon arrival animals were independently randomized into cages of 5 animals and housed 671 for an acclimatisation period of 1 week. Mice received food and water ad libitum and were 672 housed in a 12hour/12hour light dark cycle. Identification of animals within groups was 673 achieved by ear notching without anaesthesia at least 24 hours in advance of procedures. 674 Intratracheal administration of inoculum and infection

675 Anaesthesia was induced by intraperitoneal injection (BD Microlance 27G 13mm needle) 676 of $80 \mathrm{mg} / \mathrm{kg}$ ketamine and $0.8 \mathrm{mg} / \mathrm{kg}$ medetomidine. Pre-intubation body temperature was 677 maintained by contact with a heat mat (Harvard Apparatus, U.K) and eye lubricant 678 (2.0mg/g carbomer, Alcon UK) was applied. 
Intubation was achieved as previously described (Wong et al., 2019) by placement of a 21G catheter (21G IV peripheral catheter (Insyte) BD Medical) using a fibreoptic intubation set (Kent Scientific) following the enclosed instructions.

Inoculum (dose defined in graphs) was prepared by dilution of saturated overnight cultures into phosphate buffered saline (PBS) (total inoculum volume 50ul) and following inspiration it was dispersed with $2 \times 200 u$ air flushes. An aliquot of inoculum was enumerated for each administration and all inoculums were $\pm 10 \%$ of the stated figure.

Mice recovered at $32^{\circ} \mathrm{C}$ (Warm Air System, Safety Cabinet Version red, VetTech, UK) until spontaneous movement and received $0.8 \mathrm{mg} / \mathrm{kg}$ subcutaneous (BD Microlance $27 \mathrm{G}$ $13 \mathrm{~mm}$ needle) atipamezole to reverse the a-agonist into the neck scruff.

At the experimental end-point animals were anesthetized with ketamine $100 \mathrm{mg} / \mathrm{kg}$ and $1 \mathrm{mg} / \mathrm{kg}$ medetomidine by intraperitoneal injection (BD Microlance $27 \mathrm{G} 13 \mathrm{~mm}$ needles). Following the induction of anaesthesia, blood was collected by a transdiaphragmatic inferior approach cardiac puncture (BD Microlance 25G 25mm needles) and animals were then humanely killed, under anaesthesia, by cervical dislocation.

An aliquot of blood (20ul) was taken and diluted into 180ul of hypotonic lysis solution (1 $\mathrm{mM}$ EDTA in water) for enumeration of bacterial counts. The rest was placed into a microtainer (BD Microtainer for serum collection, BD Medical) and allowed to clot for 1 hour. This was then spun according to the manufacturer's guidance and aliquoted into cryobanking tubes (Greiner Bio-One) and frozen at $-80^{\circ} \mathrm{C}$ until analysis.

Lungs were dissected out, weighed and then homogenised in gentlemacs C-tubes (Miltenyi Biotec) containing $3 \mathrm{ml}$ complete RPMI media (RPMI supplemented with $10 \%$ heat inactivated FBS, $10 \mathrm{mM}$ HEPES and 1mM Sodium Pyruvate) using a gentleMac Octo dissociator (Miltenyi Biotec) using the program m_lung_1 twice. 
Bacteria were enumerated from the lung homogenate and blood by dilution in PBS and plating onto solidified LB agar containing 50ug/ml rifampicin (Merck, UK).

\section{Antibiotic delivery}

We combined the meropenem $(100 \mathrm{mg} / \mathrm{kg})$ with cilastatin $(100 \mathrm{mg} / \mathrm{kg})($ Merck, UK), a renal dihydropeptidase inhibitor, to reduce the in vivo metabolism of meropenem as the murine enzyme isoform has increased activity against this carbapenem compared to human enzyme. Drugs were diluted in $\mathrm{dH}_{2} \mathrm{O} \cdot \mathrm{dH}_{2} \mathrm{O}$ alone was administered to vehicle control animals. Doses were delivered at 6 hourly intervals as previously employed in a murine carbapenemase producing KP model (Ota et al., 2020). The drug mixture was delivered by intraperitoneal infection (BD Microlance 27G 13mm needles) to animals at 6 hourly intervals and the iliac fossa used was alternated between doses.

\section{Cytokine bead assay}

Serum IFN-Y was assessed using a custom-made mouse panel 13-plex kit (LEGENDplex, BioLegend) following the manufacturer's instructions. Cytokine levels were acquired using a FACSCalibur flow cytometer (BD Biosciences), and analyses were performed using LEGENDplex data analysis software (BioLegend). A biological repeat was considered valid only if at least $80 \%$ of all the samples run were above the limit of detection; any values below the detection limit within a repeat such as this were assumed to be the lowest value detectable by the assay for statistical analysis. Values below the detection limit in graphs were displayed as 1 for visualisation.

$\underline{\text { RNA isolation and reverse transcription quantitative PCR (RT-qPCR) }}$

$300 \mu \mathrm{l}$ of an LB overnight culture of KP were treated with RNAprotect Bacteria reagent (Qiagen) and centrifuged at $5000 \mathrm{xg}$ for $10 \mathrm{~min}$. The bacterial pellet was digested in 100 $\mu$ I TE buffer with $15 \mathrm{mg} / \mathrm{ml}$ Lysozyme (Sigma-Aldrich) and $20 \mu$ l Proteinase K (Qiagen) 
727 for 10 min according to manufacturer's guidelines. RNA was isolated using the RNeasy

728 minikit (Qiagen) following the manufacturer's instructions, and RNA concentration was determined using a Nanodrop 2000 spectrophotometer (ThermoFisher Scientific). $1 \mu \mathrm{g}$ of RNA was treated with RNase-free DNase (Sigma-Aldrich) for $1 \mathrm{~h}$ at $37{ }^{\circ} \mathrm{C}$ and cDNA was then synthesized using a Moloney murine leukemia virus (MMLV) reverse transcription kit with random primers following the manufacturer's protocol (Promega). To check for the presence of remaining DNA, a reaction mixture without the MMLV reverse transcriptase was also included (NRT). qPCR was performed using the Power Up SYBR Green master mix (ThermoFisher Scientific) and the following primers 31/32 (ompK36) and 33/34 (rpoB). The assay was run on a StepOnePlus System (Applied Biosystems) and results were analysed using the StepOne software (Applied Biosystems). Relative gene expression levels were analyzed by using the $2^{-\triangle C T}$ (where $C_{T}$ is threshold cycle) method.

\section{$\underline{\text { IVIS sfGFP fluorescence measurement }}$}

Saturated overnight cultures of strains were diluted 1:2000 in PBS. 20ul of suspension was plated onto an agar plate and allowed to dry. Following overnight incubation (16 hours exactly) plates were removed and imaged on an IVIS Spectrum CT imaging platform ( 1 second exposure, Ex 465nm/Em520nm). Fluorescence over the area of the spot was quantified using Living Image Software v5.4.3 (Perkin Elmer).

\section{Determination of RNA structures using DMS-MaPseq}

RNA structures of ompK36 $6_{\mathrm{WT}}$, ompK36 $6_{\mathrm{WT}(25 \mathrm{c}>\mathrm{t})}$ and $\mathrm{ompK} 36_{\mathrm{WT}(24 \& 25 c>t)}$ were determined using DMS-MaPseq similarly to as described previously (Zubradt et al., 2017). The principle of this method is that DMS adds methyl groups to the Watson-Crick faces of 

promoter sequence

(TAATACGACTCACTATAGGAAAAGGCATATAACAAACAGAGGG) and the reverse primer (AAGAGTATACCAGCGAGGTTAAACCGGAC). The PCR product was used as a T7 Megascript in vitro transcription (ThermoFisher Scientific) template according to manufacturer's instructions. Next, the RNA was purified using RNA Clean and Concentrator ${ }^{\mathrm{TM}}-5$ (Zymo). 10 $\mathrm{\mu g}$ RNA was denatured at $95^{\circ} \mathrm{C}$ for $1 \mathrm{~min}$. Denatured RNA was refolded by incubating in $340 \mathrm{mM}$ sodium cacodylate buffer (Electron Microscopy Sciences) and $5 \mathrm{mM} \mathrm{MgCl}{ }^{2+}$, such that the volume was $97.5 \mu \mathrm{l}$, for $20 \mathrm{~min}$ at $37^{\circ} \mathrm{C}$. Then, 2.5\% DMS (Millipore-Sigma) was added and incubated for 4 mins at $37^{\circ} \mathrm{C}$ with whole shaking at 800 r.p.m. on a thermomixer. Subsequently, DMS was neutralized by adding

$77160 \mu$ l $\beta$-mercaptoethanol (BME) (Millipore-Sigma). The DMS-modified RNA was purified 772 using RNA Clean and Concentrator ${ }^{\mathrm{TM}}-5$ (Zymo) and eluted in 10 $\mu$ l water. 
In vivo DMS modification. $500 \mu$ l of exponentially growing $E$. coli were incubated with $10 \mu l$ DMS for 3 mins at $37^{\circ} \mathrm{C}$ shaking at 800 r.p.m. on a thermomixer. DMS was quenched by adding $500 \mu \mathrm{l} 30 \%$ BME, followed by a 3 min $30 \%$ BME wash and $1 x$ PBS wash. Then, bacterial pellets were resuspended and incubated at room temperature for 5 mins in $500 \mu l$ RNAprotect $\circledast$ bacteria reagent (QIAGEN). Samples were centrifuged at $16000 \mathrm{~g}$ for 5 mins and supernatant removed. Pellets were resuspended in $100 \mu \mathrm{l}$ of $15 \mathrm{mg} / \mathrm{ml}$ lysozyme solution plus $20 \mu \mathrm{l}$ proteinase $\mathrm{K}$ and mixed by vortex for 10 seconds. Samples were incubated at room temperature for 10 mins, vortexing every 2 mins for 10 seconds. Next, samples were added with $350 \mu$ l of buffer RLT plus BME (10 $\mu$ l BME for every $1 \mathrm{ml}$ of RLT), vortex to mix. Then, $250 \mu$ l of $96-100 \%$ ethanol was added to samples. RNA was extracted using the RNeasy® Mini kit (QIAGEN) following manufacturer's instructions. DNA was digested from 5-10 $\mu$ g of total RNA per sample using the TURBO DNA-free kit (ThermoFisher Scientific) and purified using RNA Clean and Concentrator ${ }^{\mathrm{TM}}-5$ kit. Following that, ribosomal RNAs were depleted using the Ribominus ${ }^{\mathrm{TM}}$ Transcriptome Isolation kit for bacteria (ThermoFisher Scientific) following manufacturer's instructions. RNA was purified using RNA Clean and Concentrator ${ }^{\mathrm{TM}}-5$ (Zymo) and eluted in 10 $\mu$ l water.

RT-PCR and sequencing of DMS-modified RNA. To reverse transcribe, rRNA-depleted total RNA or in vitro-transcribed RNA purified from the previous steps was added to $4 \mu l$ 5x FS buffer, $1 \mu \mathrm{l}$ dNTP, $1 \mu \mathrm{l} 0.1 \mathrm{M}$ DTT, $1 \mu \mathrm{l}$ RNase Out, $1 \mu \mathrm{l} 10 \mathrm{MM}$ reverse primer (CCTGAACGTTGTATTCCC) and $1 \mu \mathrm{I}$ TGIRT-III (Ingex). The reaction was incubated for $1.5 \mathrm{~h}$ at $60^{\circ} \mathrm{C}$. Then, to degrade the RNA, $1 \mathrm{ul} 4 \mathrm{M} \mathrm{NaOH}$ was added and incubated for 3 min at $95^{\circ} \mathrm{C}$. The cDNA was purified in $10 \mu \mathrm{l}$ water using the Oligo Clean and Concentrator $^{\mathrm{TM}}$ kit (Zymo). Next, $1 \mu$ l of cDNA was amplified using Advantage HF 2 DNA 
797 polymerase (Takara) for 30 cycles according to the manufacturer's instructions (forward

798 primer: GGAAAAGGCATATAAC; reverse primer: CCTGAACGTTGTATTCCC). The PCR

799 product was purified using $\mathrm{E}-\mathrm{Gel}^{\mathrm{TM}}$ SizeSelect ${ }^{\mathrm{TM}}$ II $2 \%$ agarose gel (Invitrogen). RNA-seq

800 library for $300 \mathrm{bp}$ insert size was constructed following the manufacturer's instructions

801 (NEBNext UltraTM II DNA Library Prep Kit). The library was loaded on an iSeq-100

802 Sequencing flow cell with iSeq-100 High-throughput sequencing kit and the library was

803 run on iSeq-100 (paired-end run, 151 x 151 cycles).

804 Data analysis and visualisation

805 Metadata, including ompK36 variation, were mapped onto a phylogenetic tree of the 806 ST258/512 collection using iTOL v5.7 (Letunic \& Bork, 2021) for use in figures. An 807 interactive visualisation of this genome collection can also be accessed using Microreact v157 (Argimon et al., 2016): https://microreact.org/project/1vWbaqARPRNc55n4yfdLyQ-

809 ompk36.

810 Graphs and statistical analysis was carried out in GraphPad Prism v9.0.0 for Mac

811 (GraphPad Software, San Diego, California USA, www.graphpad.com). Data were 812 analysed for normal distribution (based on D'Agostino-Pearson or Shapiro-Wilk normality 813 tests) and if not normally distributed a base 10 logarithmic transformation was applied 814 before statistical analysis. When normality was not achieved by transformation, a non815 parametric test was applied. Statistical tests applied for each analysis are described in 816 associated figure legends.

817 Custom images of mice in Fig. 3 were made with Biorender (created with 818 BioRender.com). Images were edited and compiled into figures in Affinity Designer 819 v1.8.4. (Serif Europe Ltd). 
$821 \quad$ Primers

822 P1 GCCAGTATAGGGATAACAGGGTAATCCGGTACCGCCCGGTGTCTG

823 P2 CGCCGGATTACCCTGTTATCCCTAGGAAATCACAATTATGTTACG

824 P3 ATTACCCTGTTATCCCTATAC

825 P4 TAGGGATAACAGGGTAATCCG

826 P5 TCTGCAGTACACCCCTTC

827 P6 CATTATTTATTACCCTC

828 P7 GATTACGCCGGATTACCCTGTTATCCCTAGGCCTAATTGATTGATTAATAG

829 P8 GGCCAGTATAGGGATAACAGGGTAATAGCCCCACAGGTTGACCAGC

830 P9 GTTGCAAGCTGCATAAC

831 P10 CGCATTTGCTGCGCCTGCTAC

832 P11 GTAGCAGGCGCAGCAAATGCGCGTAAAGGCGAAGAGCTG

833 P12 GTTATGCAGCTTGCAACCGCGAAGTAATCTTTTCG

834 P13 TTAACTTTCATGTTATTAACCCTCTGTTTGTTATATG

835 P14 AGTACTGTCCCTGCTGGTACCGG

836 P15 AGTACTGTCCTTGCTGGTACCGG

837 P16 TTAACTTTCATGTTATTAACCCTCTG

838 P17 GCATATAACAAACAGAGGGTTAATAAC

839 P18 CTTTAATTATGCCACTGC

840 P19 CTTATATTATGCCACTGC

841 P20 CTATTATTATGCCACTGC

842 P21 CATTTATTATGCCACTGC

843 P22 CTTTTATTATGCCACTGC

844 P23 GCTTATAACAAACAGAGGGTTAATAAC 
P24 GCATTTAACAAACAGAGGGTTAATAAC

846

847

848

849

850

851

852

853

854

855

856

857

858

P25 GCATATTACAAACAGAGGGTTAATAAC

P26 GCATATATCAAACAGAGGGTTAATAAC

P27 GCATATAACTAACAGAGGGTTAATAAC

P28 GCATATAACATACAGAGGGTTAATAAC

P29 GCATATAACAATCAGAGGGTTAATAAC

P30 GCATATAACAAACTGAGGGTTAATAAC

P31 CCAGACCTACAACGCAACT

P32 CGCTCCAGATCCTTACCTTTAG

P33 AAGGCGAATCCAGCTTGTTCAGC

P34 TGACGTTGCATGTTCGCACCCATCA

Data availability

Raw sequence data or assemblies from the extended ST258/512 collection were published in multiple manuscripts and obtained from the European Nucleotide Archive (ENA). Genome assemblies from the larger KP collection were obtained from Pathogenwatch (https://pathogen.watch/genomes/all?genusld=570\&speciesld=573). A full list of accession numbers for all data is available in Tables S1 and S2.

\section{References}

Achouak, W., Heulin, T. and Pagès, J. M. (2001). Multiple facets of bacterial porins. FEMS Microbiol Lett 199(1), 1-7.

Adler, A., Lifshitz, Z., Gordon, M., Ben-David, D., Khabra, E., Masarwa, S., Zion, O., Schwaber, M. J. and Carmeli, Y. (2017). Evolution and dissemination of the Klebsiella 
pneumoniae clonal group 258 throughout Israeli post-acute care hospitals, 2008-13. J Antimicrob Chemother 72(8), 2219-2224.

Altschul, S. F., Gish, W., Miller, W., Myers, E. W. and Lipman, D. J. (1990). Basic local alignment search tool. J Mol Biol 215(3), 403-10.

Argimón, S., Abudahab, K., Goater, R. J. E., Fedosejev, A., Bhai, J., Glasner, C., Feil, E. J., Holden, M. T. G., Yeats, C. A., Grundmann, H., Spratt, B. G. and Aanensen, D. M. (2016). Microreact: visualizing and sharing data for genomic epidemiology and phylogeography. Microb Genom 2(11), e000093.

Argimón, S., David S., Underwood, A., Abrudan A., Wheeler, NE., Kekre, M., Abudahab, K., Yeats, C. A., Goater, R., Taylor, B., Harste, H., Muddyman, D., Feil, E. J., Brisse, S., Holt, K., Gonado-Godoy, P., Ravikumar, K. L., Okeke, I. N., Carlos, C., Aanensen, D. M. (2021). Rapid Genomic Characterisation and Global Surveillance of Klebsiella using Pathogenwatch. bioRxiv. https://doi.org/10.1101/2021.06.22.448967 Bajaj, H., Scorciapino, M. A., Moynié, L., Page, M. G., Naismith, J. H., Ceccarelli, M. and Winterhalter, M. (2016). Molecular Basis of Filtering Carbapenems by Porins from ß-Lactam-resistant Clinical Strains of Escherichia coli. J Biol Chem 291(6), 2837-47. Bankevich, A., Nurk, S., Antipov, D., Gurevich, A. A., Dvorkin, M., Kulikov, A. S., Lesin, V. M., Nikolenko, S. I., Pham, S., Prjibelski, A. D., Pyshkin, A. V., Sirotkin, A. V., Vyahhi, N., Tesler, G., Alekseyev, M. A. and Pevzner, P. A. (2012). SPAdes: a new genome assembly algorithm and its applications to single-cell sequencing. J Comput Biol 19(5), 455-77.

Berger, C. N., Crepin, V. F., Roumeliotis, T. I., Wright, J. C., Carson, D., PevsnerFischer, M., Furniss, R. C. D., Dougan, G., Dori-Bachash, M., Yu, L., Clements, A., Collins, J. W., Elinav, E., Larrouy-Maumus, G. J., Choudhary, J. S. and Frankel, G. (2017). Citrobacter rodentium Subverts ATP Flux and Cholesterol Homeostasis in Intestinal Epithelial Cells In Vivo. Cell Metab 26(5), 738-752.e6.

Bhattacharyya, S., Jacobs, W. M., Adkar, B. V., Yan, J., Zhang, W. and Shakhnovich, E. I. (2018). Accessibility of the Shine-Dalgarno Sequence Dictates N-Terminal Codon Bias in E. coli. Mol Cell 70(5), 894-905.e5.

Bowers, J. R., Kitchel, B., Driebe, E. M., MacCannell, D. R., Roe, C., Lemmer, D., de Man, T., Rasheed, J. K., Engelthaler, D. M., Keim, P. and Limbago, B. M. (2015). 
Genomic Analysis of the Emergence and Rapid Global Dissemination of the Clonal Group 258 Klebsiella pneumoniae Pandemic. Plos One 10(7).

Cassini, A., Högberg, L. D., Plachouras, D., Quattrocchi, A., Hoxha, A., Simonsen, G. S., Colomb-Cotinat, M., Kretzschmar, M. E., Devleesschauwer, B., Cecchini, M., Ouakrim, D. A., Oliveira, T. C., Struelens, M. J., Suetens, C., Monnet, D. L. (2019). Attributable deaths and disability-adjusted life-years caused by infections with antibioticresistant bacteria in the EU and the European Economic Area in 2015: a populationlevel modelling analysis. Lancet Infect Dis 19(1), 56-66.

Chiang, M. K., Lu, M. C., Liu, L. C., Lin, C. T. and Lai, Y. C. (2011). Impact of Hfq on global gene expression and virulence in Klebsiella pneumoniae. PLoS One 6(7) e22248.

Clancy, C. J., Chen, L., Hong, J. H., Cheng, S., Hao, B., Shields, R. K., Farrell, A. N., Doi, Y., Zhao, Y., Perlin, D. S., Kreiswirth, B. N. and Nguyen, M. H. (2013). Mutations of the ompK36 porin gene and promoter impact responses of sequence type 258, KPC-2producing Klebsiella pneumoniae strains to doripenem and doripenem-colistin. Antimicrob Agents Chemother 57(11), 5258-65.

Croucher, N. J., Page, A. J., Connor, T. R., Delaney, A. J., Keane, J. A., Bentley, S. D., Parkhill, J. and Harris, S. R. (2015). Rapid phylogenetic analysis of large samples of recombinant bacterial whole genome sequences using Gubbins. Nucleic Acids Res 43(3), e15.

David, S., Reuter, S., Harris, S. R., Glasner, C., Feltwell, T., Argimon, S., Abudahab, K., Goater, R., Giani, T., Errico, G., Aspbury, M., Sjunnebo, S., Feil, E. J., Rossolini, G. M., Aanensen, D. M., and Grundmann, H. (2019). Epidemic of carbapenem-resistant Klebsiella pneumoniae in Europe is driven by nosocomial spread. Nat Microbiol 4(11), 1919-1929.

Deleo, F. R., Chen, L., Porcella, S. F., Martens, C. A., Kobayashi, S. D., Porter, A. R., Chavda, K. D., Jacobs, M. R., Mathema, B., Olsen, R. J., Bonomo, R. A., Musser, J. M. and Kreiswirth, B. N. (2014). Molecular dissection of the evolution of carbapenemresistant multilocus sequence type 258 Klebsiella pneumoniae. Proc Natl Acad Sci U S A 111(13), 4988-93.

Dulyayangkul, P., Wan Nur Ismah, W. A. K., Douglas, E. J. A. and Avison, M. B. (2020). Mutation of kvrA Causes OmpK35 and OmpK36 Porin Downregulation and Reduced 
Meropenem-Vaborbactam Susceptibility in KPC-Producing Klebsiella pneumoniae. Antimicrob Agents Chemother 64(7).

Dé, E., Baslé, A., Jaquinod, M., Saint, N., Malléa, M., Molle, G. and Pagès, J. M. (2001). A new mechanism of antibiotic resistance in Enterobacteriaceae induced by a structural modification of the major porin. Mol Microbiol 41(1), 189-98.

Edgar, R. C. (2004). MUSCLE: a multiple sequence alignment method with reduced time and space complexity. BMC Bioinformatics 5, 113.

Elliott, E., Brink, A. J., van Greune, J., Els, Z., Woodford, N., Turton, J., Warner, M. and Livermore, D. M. (2006). In vivo development of ertapenem resistance in a patient with pneumonia caused by Klebsiella pneumoniae with an extended-spectrum betalactamase. Clin Infect Dis 42(11), e95-8.

EUCAST guidelines. (2021). https://www.eucast.org/clinical_breakpoints/

Fajardo-Lubián, A., Ben Zakour, N. L., Agyekum, A., Qi, Q. and Iredell, J. R. (2019).

Host adaptation and convergent evolution increases antibiotic resistance without loss of virulence in a major human pathogen. PLoS Pathog 15(3), e1007218.

Giakkoupi, P., Papagiannitsis, C. C., Miriagou, V., Pappa, O., Polemis, M., Tryfinopoulou, K., Tzouvelekis, L. S. and Vatopoulos, A. C. (2011). An update of the evolving epidemic of blaKPC-2-carrying Klebsiella pneumoniae in Greece (2009-10). J Antimicrob Chemother 66(7), 1510-3.

Giani, T., Pini, B., Arena, F., Conte, V., Bracco, S., Migliavacca, R., Pantosti, A., Pagani, L., Luzzaro, F., Rossolini, G. M. and Participants, A.-C. S. (2013). Epidemic diffusion of KPC carbapenemase-producing Klebsiella pneumoniae in Italy: results of the first countrywide survey, 15 May to 30 June 2011. Euro Surveill 18(22). Gish, W. and States, D. J. (1993). Identification of protein coding regions by database similarity search. Nat Genet 3(3), 266-72.

Gouy, M., Guindon, S. and Gascuel, O. (2010). SeaView version 4: A multiplatform graphical user interface for sequence alignment and phylogenetic tree building. Mol Biol Evol 27(2), 221-4.

Ishikawa, S. A., Zhukova, A., Iwasaki, W. and Gascuel, O. (2019). A Fast Likelihood Method to Reconstruct and Visualize Ancestral Scenarios. Mol Biol Evol 36(9), 20692085. 
Kitchel, B., Rasheed, J. K., Patel, J. B., Srinivasan, A., Navon-Venezia, S., Carmeli, Y., Brolund, A. and Giske, C. G. (2009). Molecular epidemiology of KPC-producing Klebsiella pneumoniae isolates in the United States: clonal expansion of multilocus sequence type 258. Antimicrob Agents Chemother 53(8), 3365-70.

Kohira, N., West, J., Ito, A., Ito-Horiyama, T., Nakamura, R., Sato, T., Rittenhouse, S., Tsuji, M. and Yamano, Y. (2016). In Vitro Antimicrobial Activity of a Siderophore Cephalosporin, S-649266, against Enterobacteriaceae Clinical Isolates, Including Carbapenem-Resistant Strains. Antimicrob Agents Chemother 60(2), 729-34. Kudla, G., Murray, A. W., Tollervey, D. and Plotkin, J. B. (2009). Coding-sequence determinants of gene expression in Escherichia coli. Science 324(5924), 255-8. Lam, M. M. C., Wick, R. R., Watts, S. C., Cerdeira, L. T., Wyres, K. L. and Holt, K. E. (2021). A genomic surveillance framework and genotyping tool for Klebsiella pneumoniae and its related species complex. Nat Commun 12(1), 4188. Letunic, I. and Bork, P. (2021). Interactive Tree Of Life (iTOL) v5: an online tool for phylogenetic tree display and annotation. Nucleic Acids Res 49(W1), W293-W296. Li, H. and Durbin, R. (2009). Fast and accurate short read alignment with BurrowsWheeler transform. Bioinformatics 25(14), 1754-60.

Li, H., Handsaker, B., Wysoker, A., Fennell, T., Ruan, J., Homer, N., Marth, G., Abecasis, G., and Durbin, R. (2009). The Sequence Alignment/Map format and SAMtools. Bioinformatics 25(16), 2078-2079.

Lister, P. D., Wolter, D. J. and Hanson, N. D. (2009). Antibacterial-resistant Pseudomonas aeruginosa: clinical impact and complex regulation of chromosomally encoded resistance mechanisms. Clin Microbiol Rev 22(4), 582-610.

Moore, T. A., Perry, M. L., Getsoian, A. G., Newstead, M. W. and Standiford, T. J. (2002). Divergent role of gamma interferon in a murine model of pulmonary versus systemic Klebsiella pneumoniae infection. Infect Immun 70(11), 6310-8.

Ota, K., Kaku, N. and Yanagihara, K. (2020). Efficacy of meropenem and amikacin combination therapy against carbapenemase-producing Klebsiella pneumoniae mouse model of pneumonia. J Infect Chemother 26(12), 1237-1243.

Roberts, J. A., Paul, S. K., Akova, M., Bassetti, M., De Waele, J. J., Dimopoulos, G., Kaukonen, K. M., Koulenti, D., Martin, C., Montravers, P., Rello, J., Rhodes, A., Starr, T., Wallis, S. C., and Lipman, J. (2014). DALI: defining antibiotic levels in intensive care 
995

996

997

998

999

1000

1001

1002

1003

1004

1005

1006

1007

1008

1009

1010

1011

1012

1013

1014

1015

1016

1017

1018

1019

1020

1021

1022

1023

1024

1025

1026

unit patients: are current $\beta$-lactam antibiotic doses sufficient for critically ill patients? Clin Infect Dis 58(8), 1072-83.

Rojas, L. J., Weinstock, G. M., De La Cadena, E., Diaz, L., Rios, R., Hanson, B. M., Brown, J. S., Vats, P., Phillips, D. S., Nguyen, H., Hujer, K. M., Correa, A., Adams, M. D., Perez, F., Sodergren, E., Narechania, A., Planet, P. J., Villegas, M. V., Bonomo, R. A. and Arias, C. A. (2017). An Analysis of the Epidemic of Klebsiella pneumoniae Carbapenemase-Producing K. pneumoniae: Convergence of Two Evolutionary Mechanisms Creates the "Perfect Storm". J Infect Dis 217(1), 82-92.

Seemann, T. (2014). Prokka: rapid prokaryotic genome annotation. Bioinformatics 30(14), 2068-9.

Solovyez, V. and Salamov, A. (2011). Metagenomics and its Applications in Agriculture, Biomedicine and Environmental Studies (Nova Sciences Publishers).

Tran, Q. T., Mahendran, K. R., Hajjar, E., Ceccarelli, M., Davin-Regli, A., Winterhalter, M., Weingart, H. and Pagès, J. M. (2010). Implication of porins in beta-lactam resistance of Providencia stuartii. J Biol Chem 285(42), 32273-81.

Tsai, Y. K., Fung, C. P., Lin, J. C., Chen, J. H., Chang, F. Y., Chen, T. L. and Siu, L. K. (2011). Klebsiella pneumoniae outer membrane porins OmpK35 and OmpK36 play roles in both antimicrobial resistance and virulence. Antimicrob Agents Chemother 55(4), 1485-93.

Vergalli, J., Bodrenko, I. V., Masi, M., Moynié, L., Acosta-Gutiérrez, S., Naismith, J. H., Davin-Regli, A., Ceccarelli, M., van den Berg, B., Winterhalter, M. and Pagès, J. M. (2020). Porins and small-molecule translocation across the outer membrane of Gramnegative bacteria. Nat Rev Microbiol 18(3), 164-176.

Vincent, J. L., Sakr, Y., Singer, M., Martin-Loeches, I., Machado, F. R., Marshall, J. C., Finfer, S., Pelosi, P., Brazzi, L., Aditianingsih, D., Timsit, J. F., Du, B., Wittebole, X., Máca, J., Kannan, S., Gorordo-Delsol, L. A., De Waele, J. J., Mehta, Y., Bonten, M. J. M., Khanna, A. K., Kollef, M., Human, M., and Angus, D. C. (2020). Prevalence and Outcomes of Infection Among Patients in Intensive Care Units in 2017. JAMA 323(15), 1478-1487.

Wise, M. G., Horvath, E., Young, K., Sahm, D. F. and Kazmierczak, K. M. (2018). Global survey of Klebsiella pneumoniae major porins from ertapenem non-susceptible isolates lacking carbapenemases. J Med Microbiol 67(3), 289-295. 
1027 Wong, J. L. C., Romano, M., Kerry, L. E., Kwong, H. S., Low, W. W., Brett, S. J.,

1028 Clements, A., Beis, K. and Frankel, G. (2019). OmpK36-mediated Carbapenem

1029 resistance attenuates ST258 Klebsiella pneumoniae in vivo. Nat Commun 10(1), 3957.

1030 Yoshida, K., Matsumoto, T., Tateda, K., Uchida, K., Tsujimoto, S., Iwakura, Y. and

1031 Yamaguchi, K. (2001). Protection against pulmonary infection with Klebsiella

1032 pneumoniae in mice by interferon-gamma through activation of phagocytic cells and

1033 stimulation of production of other cytokines. J Med Microbiol 50(11), 959-964.

1034 Yoshino, M., Aihara, M., Gotoh, Y., Akimoto, M., Tatsuhara, W., Kiyosuke, M.,

1035 Matsushima, Y., Uchiumi, T., Hayashi, T. and Kang, D. (2021). Stepwise Evolution of a

1036 Klebsiella pneumoniae Clone within a Host Leading to Increased Multidrug Resistance.

1037 mSphere e0073421.

1038 Zubradt, M., Gupta, P., Persad, S., Lambowitz, A. M., Weissman, J. S. and Rouskin, S.

1039 (2017). DMS-MaPseq for genome-wide or targeted RNA structure probing in vivo. Nat

1040 Methods 14(1), 75-82. 